\title{
Marriage and sexual and reproductive health of Rohingya adolescents and youth in Bangladesh: A qualitative study
}

\author{
Sigma Ainul \\ Population Council \\ Iqbal Ehsan \\ Population Council \\ Eashita Haque \\ Population Council \\ Sajeda Amin \\ Population Council \\ Ubaidur Rob \\ Population Council
}

See next page for additional authors

Follow this and additional works at: https://knowledgecommons.popcouncil.org/departments_sbsr-pgy

Part of the Demography, Population, and Ecology Commons, Family, Life Course, and Society

Commons, Gender and Sexuality Commons, and the International Public Health Commons

How does access to this work benefit you? Let us know!

\section{Recommended Citation}

Ainul, Sigma, Iqbal Ehsan, Eashita Haque, Sajeda Amin, Ubaidur Rob, Andrea J. Melnikas, and Joseph Falcone. 2018. "Marriage and sexual and reproductive health of Rohingya adolescents and youth in Bangladesh: A qualitative study," research report. Dhaka: Population Council. 


\section{Authors}

Sigma Ainul, Iqbal Ehsan, Eashita Haque, Sajeda Amin, Ubaidur Rob, Andrea J. Melnikas, and Joseph Falcone 


\section{MARRIAGE AND SEXUAL AND REPRODUCTIVE HEALTH OF ROHINGYA ADOLESCENTS AND YOUTH IN BANGLADESH: A QUALITATIVE STUDY}

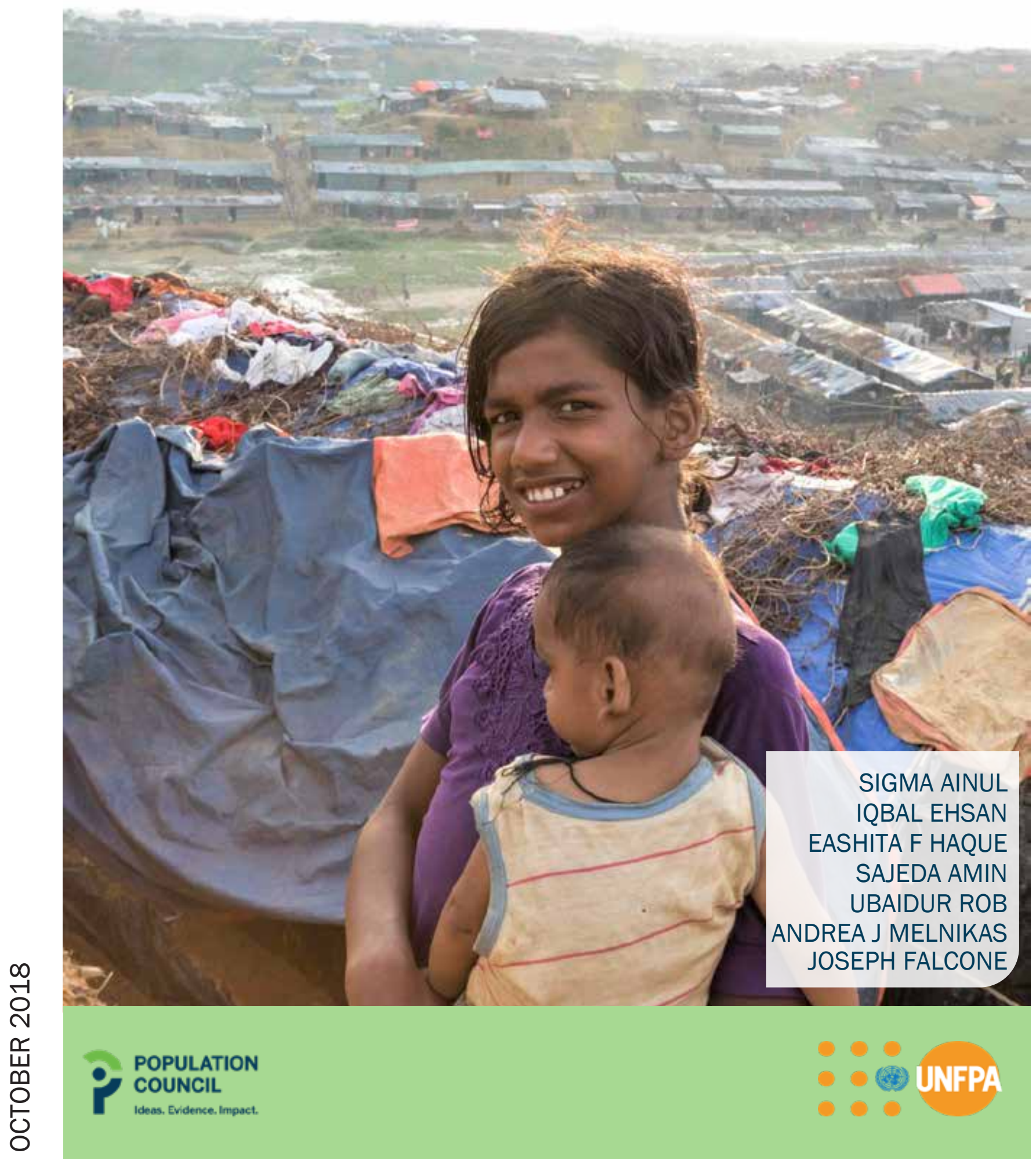





\section{Population P council \\ Ideas. Evidence. Impact.}

The Population Council confronts critical health and development issues-from stopping the spread of HIV to improving reproductive health and ensuring that young people lead full and productive lives. Through biomedical, social science, and public health research in 50 countries, we work with our partners to deliver solutions that lead to more effective policies, programs, and technologies that improve lives around the world. Established in 1952 and headquartered in New York, the Council is a nongovernmental, nonprofit organization governed by an international board of trustees.

Population Council

House 12, Road 25/30

Gulshan, Dhaka 1212

Bangladesh

popcouncil.org

This study has been funded by UNFPA.

Suggested citation: Ainul, Sigma, Iqbal Ehsan, Eashita F. Haque, Sajeda Amin, Ubaidur Rob, Andrea J. Melnikas, and Joseph Falcone. 2018. "Marriage and Sexual and Reproductive Health of Rohingya Adolescents and Youth in Bangladesh: A Qualitative Study.” Population Council: Dhaka, Bangladesh.

Cover and back cover photo credit: Faiham Ebna Sharif

@ 2018 The Population Council, Inc. 


\section{Table of Contents}

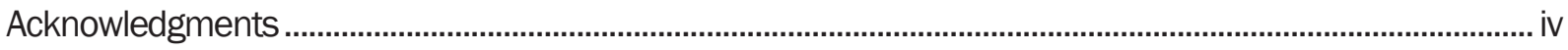

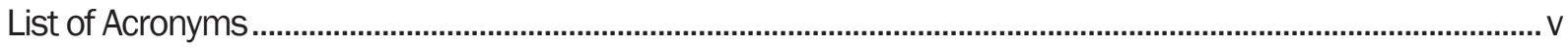

Executive Summary ..................................................................................................................................

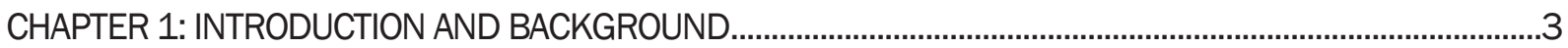

CHAPTER 2: METHODOLOGY

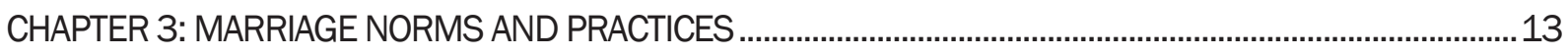

CHAPTER 4: FAMILY PLANNING AND CHILDBEARING NORMS AND PRACTICES ......................................17

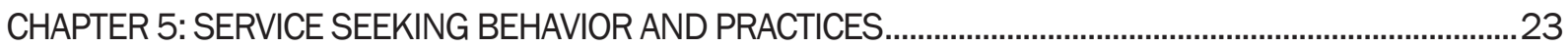

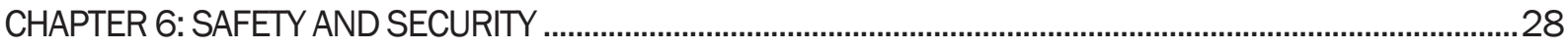

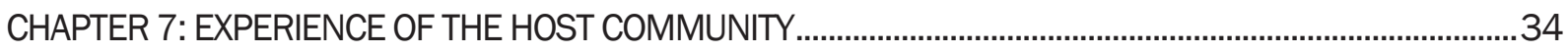

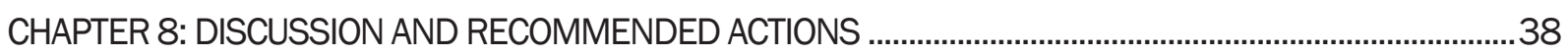

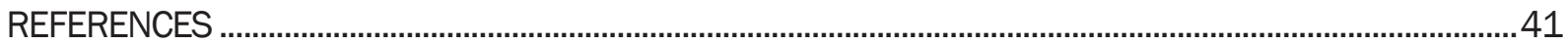




\section{Acknowledgments}

This study has been conducted as part of a larger project "Accelerating access to end child marriage in Bangladesh" led by the Population Council with financial support from UNFPA. We also gratefully acknowledge the support of two individual donors, Jerry Cunningham and Diane Cunningham, for their financial contribution to this study.

The authors wish to acknowledge the contributions of the many individuals and institutions who made this study possible. We are grateful to the UNFPA in Bangladesh for their generous support, in particular Sathyanarayanan Doraiswamy, Eshani Ruwanpura, and Mahboob E Alam, for their valuable insight during the development of the study protocol and finalization of the report.

Special thanks are due to Mohammad Abul Kalam NDC (Additional Secretary), Refugee Relief \& Repatriation Commissioner, for ensuring our access to the Rohingya camps for data collection. We are also thankful to Md. Abdus Salam, Civil Surgeon, Cox's Bazar, for his cooperation throughout the study.

We are thankful to Irfan Hossain from the Bangladesh office for his valuable contribution and assistance during the development of the study protocol. We also thank Dipak Kumar Shil for managing the study funding, budget, and expenditure reports. We thank Mamun-or-Rashid and Joynal Abedin, who provided day-to-day administrative support for this study.

In addition, we would like to convey our sincere thanks to our seven research assistants, Tasnoba Azam, Tahera Banu, Tuhiratun Nesa, Ching U Pru, Arpon, Kumer Das, Sakib Hosain and Bana Ratna Tanchangya for their hard work during the data collection and transcription periods. We are also thankful to our six Rohingya youths for their support in creating an enabling environment for the data collection team and helping them access Rohingya respondents.

Finally, a special thank you goes to our study respondents who shared their experiences and perspectives with us, without which this study would not have been possible. 


\section{List of Acronyms}

\begin{tabular}{|c|c|}
\hline ACAPS & Assessment Capacities Project \\
\hline AIDS & Acquired Immune Deficiency Syndrome \\
\hline ANC & Antenatal Care \\
\hline $\mathrm{CiC}$ & Camp in Charge \\
\hline DGHS & Directorate General of Health Services \\
\hline FGD & Focus Group Discussion \\
\hline $\mathrm{FP}$ & Family Planning \\
\hline GO & Government Organization \\
\hline GBV & Gender-based Violence \\
\hline HIV & Human Immunodeficiency Virus Infection \\
\hline icddr,b & International Centre for Diarrhoeal Disease Research, Bangladesh \\
\hline IDI & In-depth Interview \\
\hline INGO & International Non-governmental Organization \\
\hline ISCG & Inter Sector Coordination Group \\
\hline IRB & Institutional Review Board \\
\hline MOHFW & Ministry of Health and Family Welfare \\
\hline NDC & National Defence College \\
\hline NGO & Non-governmental Organization \\
\hline NPM & Needs and Population Monitoring \\
\hline PNC & Postnatal Care \\
\hline RA & Research Assistant \\
\hline $\mathrm{RH}$ & Reproductive Health \\
\hline RRRC & Refugee Relief and Repatriation Commissioner \\
\hline SRH & Sexual and Reproductive Health \\
\hline STI & Sexually Transmitted Infections \\
\hline UNFPA & United Nation Population Fund \\
\hline UNHCR & United Nations High Commissioner for Refugees \\
\hline UNICEF & United Nations International Children's Fund \\
\hline WHO & World Health Organization \\
\hline
\end{tabular}




\section{Executive Summary}

The Rohingya have been fleeing from Myanmar's Rakhine State to adjacent districts in Bangladesh for decades. The latest influx began in August 2017 and has led to a fourfold increase in the Rohingya population in Bangladesh. It is estimated that over one million Rohingya are now living in Bangladesh in Rohingya camps located in two upazilas (Ukhia and Teknaf) of Cox's Bazar, Bangladesh. UNFPA is leading response efforts for the sexual and reproductive health needs of women and adolescent girls in this crisis.

Due in part to a history of exclusion in Myanmar, the Rohingya have not been counted in censuses and very little is known about their basic socio-demographic characteristics including health, education, marriage patterns, maternal and sexual and reproductive health knowledge, and their service seeking behavior. In 2018, with support from UNFPA, icddr,b conducted a survey to provide basic demographic information as part of a needs assessment of maternal and child health among women of reproductive age living in camps. The current qualitative study complements the quantitative survey with specific emphasis given to the needs of Rohingya adolescents and youth. The sample included 48 married and unmarried Rohingya adolescent girls and boys aged between 14-24 years, 48 community and religious leaders, 20 adult Rohingya women, 24 program managers and service providers working with Rohingya populations in Bangladesh, and 53 adolescents and young people from the host community. This research study is not designed to establish prevalence, or to provide a quantitative or objective account of the experiences of Rohingya adolescents and youth, but rather to draw out and amplify the voices of Rohingya adolescents and youth and provide a rich understanding of the experiences and needs of adolescent and youth in crisis.

While all displaced Rohingya face numerous challenges related to their displaced status, adolescents and youth are affected by this protracted crisis in ways that are different from the adult population, and in ways that may be overlooked. The current study focuses specifically on displaced Rohingya adolescents and youth (ages 14-24) and explores how the crisis has impacted their sexual and reproductive health (SRH), marriage practices, safety and security, and related attitudes and aspirations. This research aims to identify approaches to improve adolescents' knowledge of SRH issues and their access to and uptake of services, and to identify gaps in programming knowledge and practice.

\section{Key Findings}

\section{Child marriage is practiced among the Rohingya}

Participants reported a clear preference for child marriage. In the absence of well-defined laws regarding minimum age at marriage and marriage registration, child marriages are on the rise. Our discussions indicate the informality of marriage practices enabled by the absence of rules in camps stand in sharp contrast to the severe restrictions and high fees imposed by Myanmar administrators and enforced by military personnel in Myanmar. There is an absence of legal processes of documentation of marriage in camps because of the ambiguous status of the Rohingya in Bangladesh; this absence allows the Rohingya more perceived control over marriage in their communities in Bangladesh than they had in Myanmar.

\section{Arranged marriages and dowry exchange are the norm among the Rohingya}

Marriages are arranged between families and girls are considered marriageable after they reach puberty. There are strong beliefs that Islam mandates marriage soon after puberty for girls. These beliefs are reinforced by the fear of sexual violence and/or involvement of girls in "illicit" sexual relationship before marriage. Exchange of dowry is traditionally practiced among the Rohingya in Myanmar and it is still prevalent even in the constrained economic condition in the camps. Respondents expressed concern about not having enough marriageable Rohingya men in the camps but denied that Rohingya girls are marrying Bangladeshi men. There is a general awareness of laws prohibiting such marriages.

\section{Large family size is preferred and use of contraception is limited among the Rohingya}

The Rohingya respondents believe that Islam prohibits contraception and encourages pronatalism. Among the Rohingya, sentiments such as "women are born to bear children" and trying to limit the number of children is a "sin" are common. Religious leaders play a major role in shaping perception and practices in this community. Children are considered to be economic assets and a "gift of Allah" who bring prosperity to the family. The use of contraception is limited and reinforced by beliefs that the use of family planning 
methods is associated with immoral behaviors. One common misperception among the Rohingya is that contraception use may cause infertility and even death. This was repeated by elders in the community. Respondents reported that husbands and mothers-in-law are the two most important actors in decisionmaking regarding contraceptive use and reproductive health service seeking behavior for the women and girls. Knowledge about STI and HIV was limited among the Rohingya, and misinformation about reasons for spreading HIV was also prevalent.

\section{Home delivery used to be the widespread practice among the Rohingya in Myanmar and is still in} practice in the camps

In the rural areas of Rakhine state, there are few public health facilities and services; this, coupled with limited mobility for the Rohingya, impedes access to health services for pregnant women. Pregnant women also have limited knowledge about ANC and PNC services. Participants reported that home delivery by local birth attendants was a common practice among the Rohingya. There was a sense of mistrust among the Rohingya about service providers in Myanmar and misconceptions that children will die if they go to hospitals in Myanmar.

\section{Reproductive health services are often available but inaccessible for the Rohingya adolescents and youth in the camps}

Despite the availability of health care services in the camps, the service uptake for maternal and sexual reproductive health is limited, mostly due to a long-standing practice of restricted mobility for girls and women outside of the home. Issues with poor road conditions, barriers to transportation, and shyness among adolescents were also reported by service providers as reasons for limited service uptake among Rohingya adolescents. Service providers identified outreach as an effective strategy for reaching them.

Despite issues, the Rohingya consider living in camps safer than living in Myanmar

Despite the fact that living in camps can be stressful due to cramped conditions, limited access to resources, and restricted mobility within demarcated areas, we found that the Rohingya feel life in the camp is much safer compared to their lives in Myanmar. Respondents denied knowledge of cases of trafficking or forced/transactional sex and emphasized the fact that the Rohingya maintain religious order and refrain from getting involved in any wrongdoing.

\section{The host community perceives the Rohingya as both social and economic threats}

In speaking to individuals in a host community, we found that perceptions of the social lives of the Rohingya were quite different. In FGDs with the host community, we found reports of high tension between the host community and the Rohingya. Although the host community acknowledged being sympathetic towards the Rohingya early on, gradually this empathy has eroded as the presence of the Rohingya in the region has disrupted their normal lives and routines. In FGDs, the host community reported incidents of violence and the involvement of young Rohingya boys in smuggling and drug peddling. Members of the host community also allege that Rohingya girls are involved in transactional sex with Bangladeshi men in and around the camp. They blame the Rohingya for an increase in polygamy, familial conflicts, and violence in the host community. Members of the host community also shared their concerns about environmental degradation through deforestation and limited livelihood options, increased food prices, and transportation problems they perceive as associated with an influx of Rohingya refugees. We heard from the host community that they fear being outnumbered by the Rohingya and being neglected by NGOs and service providers. 


\section{CHAPTER 1: INTRODUCTION AND BACKGROUND}

\section{INTRODUCTION}

Since August 2017 the influx of over 720,000 Rohingya to Bangladesh has created the fastest growing refugee crisis in the world. Over one million displaced Rohingya now reside in camps located in two upazilas (Ukhia and Teknaf) of Cox's Bazar, Bangladesh. The vast majority are women and children (UNFPA 2018; ISCG 2018; IOM 2018a; IOM 2018b) and almost 60 percent of the population are under the age of 18; the crisis has therefore been described as a children's emergency (Save the Children 2018).

The Bangladesh Government and development partners such as UNHCR, UNICEF, UNFPA, WHO, as well as several national and international development organizations are working jointly to provide humanitarian assistance to the Rohingya people. UNFPA is supporting response efforts to address the unique health and protection needs of women and adolescent girls caught in this crisis. Although there are sexual and reproductive health (SRH) services available in the camps, providers report that adolescents and youth do not often access these services. There is a need for reliable data to better understand existing service demands and barriers to access in order to serve the current population and plan for the protracted situation ahead.

Historically, the Rohingya population has been understudied and undercounted due to a lack of formal recognition by the Myanmar Government, leading to their exclusion from the national census (Mahmood 2017; Myanmar Population and Housing census, 2014). There is limited information available about this population's socio-demographic profile, basic health status, education or livelihood experiences, marriage patterns, child bearing practices, family planning behaviors, maternal health and SRH knowledge and service-seeking behaviors.

In an attempt to fill the evidence and knowledge gap, iccdr,b with support from UNFPA conducted a study "Demographic profiling and needs assessment of maternal and child health care for Rohingya refugee population in Cox's bazar, Bangladesh" (Chowdhury et al. 2018) in 2018. This study assessed the current state of the Rohingya population in Bangladesh with special attention given to pregnancy, lactation, and family planning, and to the health status of children under 5 years. The icddr,b study employed a cross-sectional quantitative study design and collected information from 11 Rohingya camps located in Ukhia and Teknaf upazilas. Data were collected from women of reproductive age (13-49 years), caregivers of under-5 children, and household heads. Importantly, this study presented demographic profiles (age, sex, education, marital status, birth rate, death rate, and household size) for over three thousand households consists of sixteen thousand Rohingya individuals. As part of this study, over three thousand women of reproductive age (13-49 years) were interviewed about their reproductive and birth history, family planning method uses and choices, antenatal care (ANC) history, recent delivery experiences, and lactation history. This study also assessed current illness, handwashing practices, and satisfaction with regards to services including housing/shelter, sanitation, and health care. 
Box 1: Empirical evidence from the icddr'b study on SRH and FP among the Rohingya

The study included 16,588 individuals from 3050 households with a mean household size of $5.3 \pm$ 2.3. Among the study population, $45.8 \%$ were 13 49 years of age. The study found similar age-sex distribution in the Rohingya population residing in registered old camps and the new arrivals. About $70 \%$ of the women of reproductive age (13-49 years) were below 30 years.

\section{Marriage and child bearing}

The age at marriage was $16.8 \pm 2.2$ years and the reported mean age at first pregnancy was $18.0 \pm$ 2.4 years, suggesting that child marriage and early pregnancy are common among the Rohingya.

\section{Contraceptive use}

About $86 \%$ of currently married women [N=2,227] had heard of at least one method of family planning. The contraceptive prevalence rate (CPR) was about 34\%. Injection (Depo-Provera) (70.5\%) and oral contraceptives (28.9\%) were the two most popular FP methods reported by current contraceptive users.

\section{Facility delivery}

Among those surveyed, $14.0 \%$ of ever married women $(n=370)$ were currently pregnant. Regarding plans for delivery, $10 \%$ reported being willing to deliver at a health facility, and about 36\% reported wanting to deliver their child at home. The majority (54\%) reported that they had not yet decided on their delivery plan.
Although the icddr,b study generated useful data about the Rohingya population's family size, family planning, and maternal health service seeking behavior and practices, still more information is needed to adequately describe and provide effective services for this population. To reach this population effectively with programs, we need to further explore the underlying causes of their practices and understand the interplay of nuanced social dynamics of the Rohingya population that influence their behavior and practices.

This current qualitative study builds on and complements the quantitative study conducted by icddr,b to explore the relevant issues more in-depth. It provides useful context and understanding to explain practices and behaviors among the Rohingya population and recommend actions for effective programmatic interventions to more appropriately meet their needs.

While the icddr,b study focused on pregnant and lactating women of reproductive age (13-49 years), the current qualitative study focuses on the sexual and reproductive health $(\mathrm{SRH})$ needs of Rohingya adolescents and youth. It also explores marriage practices and patterns among the Rohingya

people, because in the context of South Asian culture, marriage marks the beginning of sanctioned sexual activity and thus holds special importance in studying SRH and related outcomes for girls and women. Because child marriage is associated with a range of $\mathrm{SRH}$ outcomes such as, early, mistimed, and/or unwanted pregnancy, poor knowledge and access to family planning and maternal health services, unsafe sex, increased risk of sexually transmitted infections (STIs) and HIV (Bruce and Clark 2004; Santhya and Jejeebhoy 2007; Santhya 2011; Ainul and Amin 2015, Ainul et. al 2017), particular attention is paid to child marriage in this setting.

To understand how and to what extent SRH and marriage practices have changed due to displacement, the current qualitative study enquires about practices in this population during two distinct periods in time: pre- and post- arrival in Bangladesh. This study also includes participants from the surrounding host community to help understand opportunities and constraints presented in the context of displacement and the acceptance of the receiving community.

The Population Council, with financial support from UNFPA, conducted this qualitative assessment to describe SRH needs of Rohingya adolescents and youth living in Ukhia upazila, Cox's Bazar district, Bangladesh to generate useful knowledge for improving and strengthening the SRH program moving forward. 
The key objectives of the study were:

- To assess SRH needs of Rohingya adolescents and youth and the extent to which existing Service Delivery Points (SDPs) address them.

- $\quad$ To assess the availability and quality of SRH services provided by SDPs to Rohingya adolescents and youth.

- To identify gaps and challenges from demand and supply end that can be used to design the provision of SRH services to this vulnerable group in more effective ways.

The study has also explored the Rohingya population's marriage and childbearing rituals and practices (in Myanmar and in camps in Bangladesh), their experience of abuse and sexual violence, substance abuse, and their future aspirations. This report presents major findings from the qualitative study and is intended to inform policy makers and program managers about the special needs of Rohingya adolescents and youth living in camps.

\section{HISTORICAL BACKGROUND}

The Rohingya are a Muslim ethnic minority group that have been persecuted for generations and forcefully displaced due to increasing violence in their place of origin in the Northern Rakhine state of Myanmar. According to the Myanmar Citizenship Law of 1982, Rohingya Muslims are considered to be "stateless" and "illegal immigrants" (UNHCR 2017). The process of "othering," discriminatory treatment, and "ethnic cleansing" of the Rohingya people in Myanmar is well documented and goes back for decades (Mahmood et. Al. 2017; Sen 2008; Medecins Sans Frontieres 2013). The Rohingya have faced a continuing situation of severe and systemic oppression characterized by the lack of freedom of movement, limited access to sufficient food, inadequate health care, and restricted educational and livelihoods opportunities (ACAPS 2017, OHCHR 2018).

Notably, institutionalized discrimination against Rohingya minorities in Myanmar is reflected through restrictions that the state imposes on marriage and birth registration for Rohingya (Hickox 2009). In order to register marriages, the Myanmar government required Rohingya to obtain licenses through military authorities, a process which usually took years to complete, and required identity checks and a large sum of money (Mahmood et al. 2017). Travel restrictions for the Rohingya severely limit their access to maternal and child health care at the government health clinics in Myanmar (Myo 2013; Sheehy et al. 2015; Mahmood et al. 2017).

For decades, the healthcare needs of the Rohingya have been severely neglected by Myanmar's government, a situation that has been exacerbated by violence and retribution against them. Rakhine is among the least-developed areas of Myanmar and access to healthcare across the state is generally very poor (OHCHR 2018). According to the report of the Advisory Commission on Rakhine State, there are only five health workers per 10,000 people, which falls well below the national average of 16 per 10,000 people, and even further below the minimum of 23 per 10,000 people recommended by the WHO to maintain a functional health system (OHCHR 2018; Amnesty 2017). The healthcare situation worsened from 2012, following a wave of violence in Rakhine and the government's explicit exclusion of the Rohingya from the better-equipped health care facilities such as the Sittwe General Hospital (OHCHR 2018). Humanitarian agencies have been the primary healthcare providers for Rohingya for many years (UNHCR 2018; Amnesty 2017; Mahmood et Al. 2017), however, the activities of these organizations were often limited by government-imposed restrictions such as the requirement that international humanitarian staff obtain travel authorization for movement within the Rakhine State.

The discriminatory treatment and persecution of the Rohingya in Myanmar has caused mass displacement, both within the country and across international borders. Bangladesh has a long history of hosting displaced Rohingya with the earliest arrivals recorded in 1948 (UNHCR 2007). An influx occurred in 1991-92 when over 250,000 Rohingya fled to Bangladesh in the wake of serious state repression in 
Myanmar. Another massive influx began on 25 August 2017, after a Myanmar military crackdown in northern Rakhine state led around 720,000 Rohingya to flee to Bangladesh (ISCG 2018). This latest migrant influx has resulted in a total Rohingya of over one million in Bangladesh. The majority of the incoming Rohingya is housed in two upazilas of Cox's Bazar district: Ukhia and Teknaf. The rapid population increase in these areas has placed extensive pressure on local infrastructure and resources.

\section{THE CURRENT HEALTH SITUATION IN ROHINGYA COMMUNITIES IN BANGLADESH}

The Government of Bangladesh and its development partners coordinated the efforts to provide protection and assistance to the displaced Rohingya. Government of Bangladesh has allocated 6,000 acres of land to host the Rohingya in Ukhia and Teknaf (RRRC and ISCG 2018). In Ukhia, the Rohingya population is now four times the local Bangladeshi population. The following table presents the distribution of the Rohingya and Bangladeshi population in Cox's Bazar.

Table 1: Distribution of Rohingya and Bangladeshi population in Cox's Bazar

\begin{tabular}{llllll}
\hline & Cox's Bazar Sadar & Ramu & Teknaf & Ukhia & Total \\
Rohingya population & 5,393 & 1,590 & 184,679 & 726,901 & 918,563 \\
Bangladeshi citizens & 522,435 & 266,640 & 263,689 & 155,187 & $1,207,951$ \\
\hline
\end{tabular}

As foreigners, the Rohingya do not have permission to work in Bangladesh because the country is not signatory to the 1951 convention relating to the status of Refugees. The Rohingya's ability to pursue livelihoods is restricted, and they are completely dependent on humanitarian assistance for meeting their basic needs including water, food, shelter, and health services (UNHCR 2018; UNICEF 2018).

To address this emergency situation, 150 national and international development partners are engaged in provision of health services in the refugee camps in Ukhia, and Teknaf and in reinforcing existing health facilities for a strengthened public health system more broadly (WHO 2018). While some partners are providing a minimal initial package of SRH services, access to essential reproductive, maternal, and newborn health services remains a major challenge. The Directorate General of Health Services (DGHS) under the Ministry of Health and Family Welfare (MOHFW) is working with the Bangladesh Armed Forces Division, UN agencies, and international and national NGOs to provide health services in Rohingya camps.

Overall, the health sector partners are coordinated under the leadership of the Civil Surgeon's Office of Cox's Bazar, the DGHS Coordination Centre, RRRC, and WHO for better planning and implementation of emergency response. Together, 107 partners have established a total of 163 basic health units, 33 primary health centers, and 11 hospitals to provide comprehensive and life-saving reproductive, maternal, neonatal, and adolescent health care to the Rohingya people living in camps (ISCG 2018). 


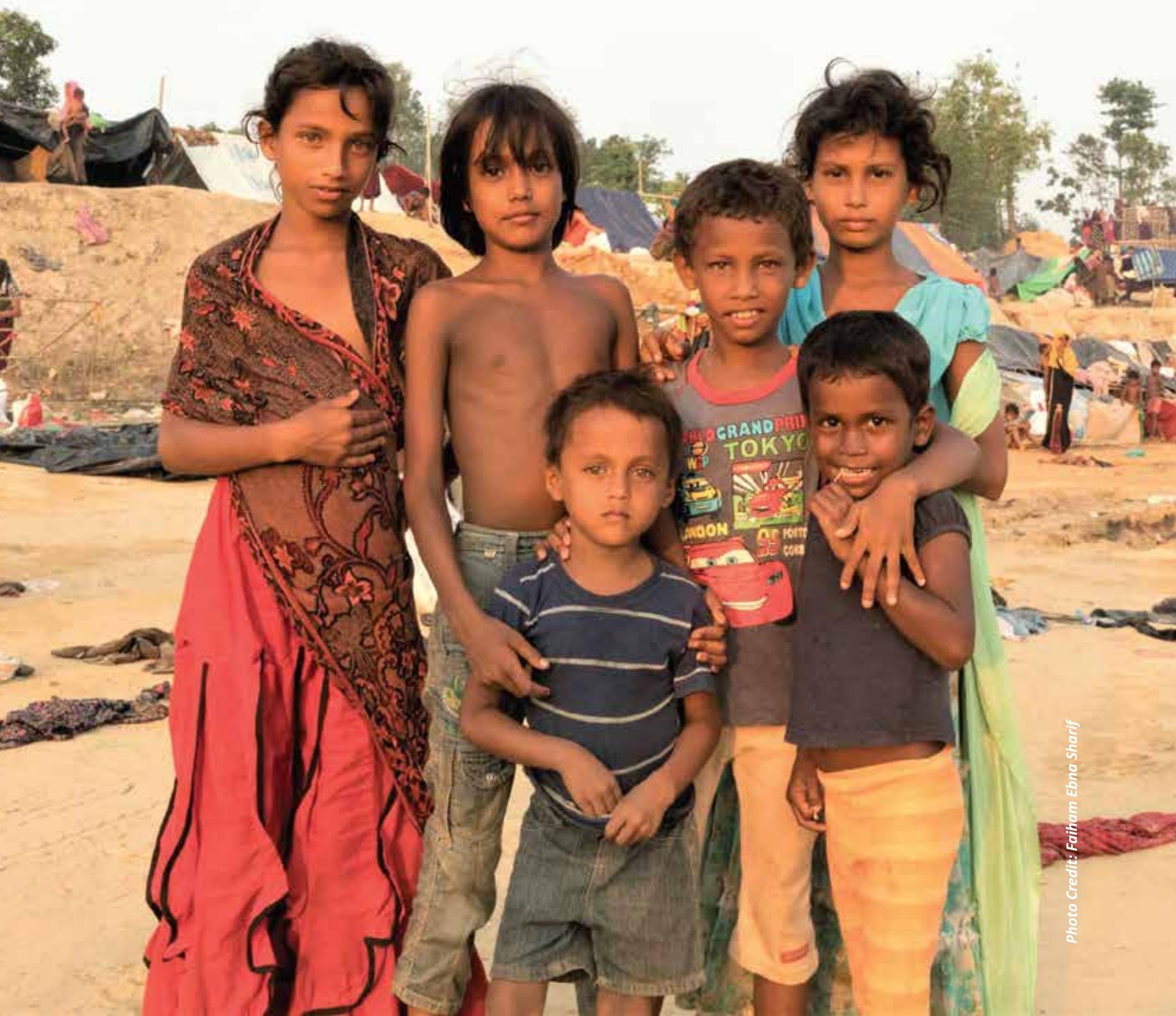




\section{VULNERABILITY OF ADOLESCENTS IN THE ROHINGYA REFUGEE CRISIS}

In Rohingya refugee settlements-as is the case in other humanitarian emergency settings-children, adolescents, and women are at the highest risk for numerous negative outcomes. Many children and adolescents are separated from their parents, families, and communities during the displacement process and face increased risk of poverty, critical illness, violence, sexual exploitation, and abuse (UNFPA \& Save the Children 2009). Unaccompanied children and adolescent populations in humanitarian crisis settings have been found to have greater psychiatric morbidity than the general population (Huemer et al. 2009).

The number of Rohingya women and girls having experienced rape and sexual abuse in Myanmar or Bangladesh is unknown, but it is thought to be high because as with other humanitarian crises, it is known that women and girls are at an increased risk of sexual and gender-based violence, sexually transmitted infections (STIs) including HIV, unintended pregnancy, and maternal death and illness. Over 2,000 GBV cases have been reported through November 2017 (ISCG 2017) and women and girls in refugee camps are also reported to have engaged in survival sex (UN WOMEN 2017; CARE 2017). Global evidence shows that girls especially, but also boys under the age of 18 , often make up the majority of survivors of sexual violence in conflict and conflict-affected countries; sometimes more than 80 percent of those affected by sexual violence are children (Save the Children 2013).

Child marriage is also another issue affecting girls during a crisis situation. Refugee crises are characterized by the loss of livelihoods, decreased economic opportunity, heightened insecurity, and the absence of education, all of which can contribute to changes in marriage patterns and practices and increases in child marriage (Mourtada et al. 2017; Girls not brides 2016). Although child marriage is often perceived as a means of protecting the economic and physical security of girls, marriage at young ages can pose multiple threats to young girls' lives, health, and future prospects. Complications during pregnancy and delivery are the second leading cause of death among 15- to 19-year-olds and 90 percent of early first births happen within the context of child marriage (WHO 2016). Pregnant adolescents are at increased risk of obstructed labor, a life-threatening obstetric emergency that can develop due to the immature pelvis that is too small to allow the passage of a baby through the birth canal (UNFPA and Save the Children 2009). There are strong possibilities for obstetric fistula or uterine rupture, hemorrhage, and death of the mother and child if treatment is delayed. However, during such crisis situations, emergency obstetric care services are often unavailable, increasing the risk of morbidity and mortality among adolescent mothers and their babies (UNFPA and Save the Children 2009). Globally, 60 percent of preventable maternal deaths take place in settings of conflict, fragility, displacement, and natural disasters (WHO 2016).

In the Rohingya crisis, as in other complex humanitarian disasters, access to SRH information and services is a critical issue for women and adolescents. A multi-country baseline study on displaced population settings by Women's Refugee Commission suggests that awareness of family planning and contraceptive use is generally lower in refugee camps than in surrounding settlements. UN Women found that the awareness and acceptance of long-acting and permanent family planning methods are particularly low in refugee camp settings (UN Women 2017). Compared with married adolescents, unmarried adolescents face even greater barriers in terms of access to information and services on contraceptive methods. Adolescent girls' needs and vulnerabilities vary based not only on the social and gender dynamics that constrict their lives and infringe upon their rights, but also on how humanitarian sectors respond (or do not respond) to their specific needs, risks, and disadvantages (Women's Refugee Commission 2016). 


\section{CHAPTER 2: METHODOLOGY}

\section{RESEARCH DESIGN}

The study used qualitative methodology to collect information from a specific group of respondents through in-depth interviews (IDI) and focus group discussions (FGD). The sample included married and unmarried Rohingya adolescent girls and boys aged between 14-24 years ${ }^{1}$, community leaders (Majhee ${ }^{2}$, Imams ${ }^{3}$ ), adult Rohingya women, service providers and program personnel from public sector, development organizations, and national and international NGOs working with Rohingya populations in Bangladesh. Adolescents and young people from the host community were also included in the study as respondents primarily to assess local attitudes towards the Rohingya. Purposive sampling technique was used to select study participants. Table 2 describes the number ${ }^{4}$ of IDIs and FGDs according to different categories of respondents:

Table 2: Sample size by data collection method

\begin{tabular}{|c|c|c|c|c|c|c|}
\hline & $\begin{array}{l}\text { IDI with Rohingya } \\
\text { population }\end{array}$ & $\begin{array}{l}\text { IDI with } \\
\text { Program } \\
\text { managers }\end{array}$ & $\begin{array}{l}\text { IDI with } \\
\text { Service } \\
\text { providers }\end{array}$ & $\begin{array}{l}\text { FGD with } \\
\text { Majhee \& } \\
\text { Imam }\end{array}$ & $\begin{array}{l}\text { FGD with } \\
\text { Rohingya } \\
\text { women }\end{array}$ & $\begin{array}{l}\text { FGD with host } \\
\text { community }\end{array}$ \\
\hline $\begin{array}{l}\text { Sample size } \\
\& \text { study } \\
\text { population }\end{array}$ & $\begin{array}{l}48 \\
\text { Female (12 Married } \\
\& 12 \\
\text { Unmarried) } \\
\text { Male (12 Married \& } \\
12 \text { Unmarried) }\end{array}$ & $\begin{array}{l}12 \\
\text { (NGOs, INGOs, } \\
\text { UN, DGHS) }\end{array}$ & $\begin{array}{l}12 \\
\text { (6 Doctors } \\
\text { and } 6 \\
\text { Paramedics/ } \\
\text { frontline } \\
\text { workers) }\end{array}$ & $\begin{array}{l}4 \\
\text { Majhees (2) } \\
\text { Imams (2) }\end{array}$ & $\begin{array}{l}2 \\
\text { Adults } \\
\text { Female }\end{array}$ & $\begin{array}{l}6 \\
\text { Female (4) } \\
\text { Male (2) }\end{array}$ \\
\hline
\end{tabular}

Data collection tools were developed between the months of May and June 2018. Data collection and transcription were completed during the month of July and August 2018. Data analysis and report writing were completed in September 2018.

\section{STUDY LOCATIONS AND POPULATION CHARACTERISTICS}

According to the latest Needs and Population Monitoring (NPM) Site Assessment the majority of the Rohingya refugees live in Ukhia upazila (comprising 80 percent of the total households) and the remaining lives in Teknaf (comprising 20 percent of households) (IOM Bangladesh 2018b). Data were collected from Balukhali- Kutupalong expansion site and the surrounding host community of Palongkhali Union of Ukhia upazila.

The entire camp area is organized into twenty zones (also called camps). Out of these twenty camps, five camps were selected based on their geographical locations, to cover different parts (East, West, South, North and Center) of the larger camp area (See Figure 1). Data were collected from camps 4, 8E, 10, 15, and 20. Within each camp, eligible individuals were selected randomly for interview (see eligibility criteria below). Research Assistants stationed in Cox's Bazar town traveled to Ukhia for data collection by car. It took about 60-80 minutes to reach the field locations.

\footnotetext{
${ }^{1}$ Although UN definition of adolescents and youth are those between 10-24, we have purposively excluded adolescents aged below 14 to preserve their best interest. Topics of the interview, level of physical and mental maturity and prior experiences of the child (possible traumatized experience during their displacement) were taken into considerations in excluding young adolescent.

2 The Majhee's main tasks are supporting the Bangladesh army and humanitarian agencies in organizing aid distribution processes and maintaining communications with the Rohingya community living in the camps.

3 Imams are the religious leaders who play a crucial role in shaping religious norms and beliefs among the Rohingya

${ }^{4}$ Time, budget and saturation of findings guided the sample size
} 


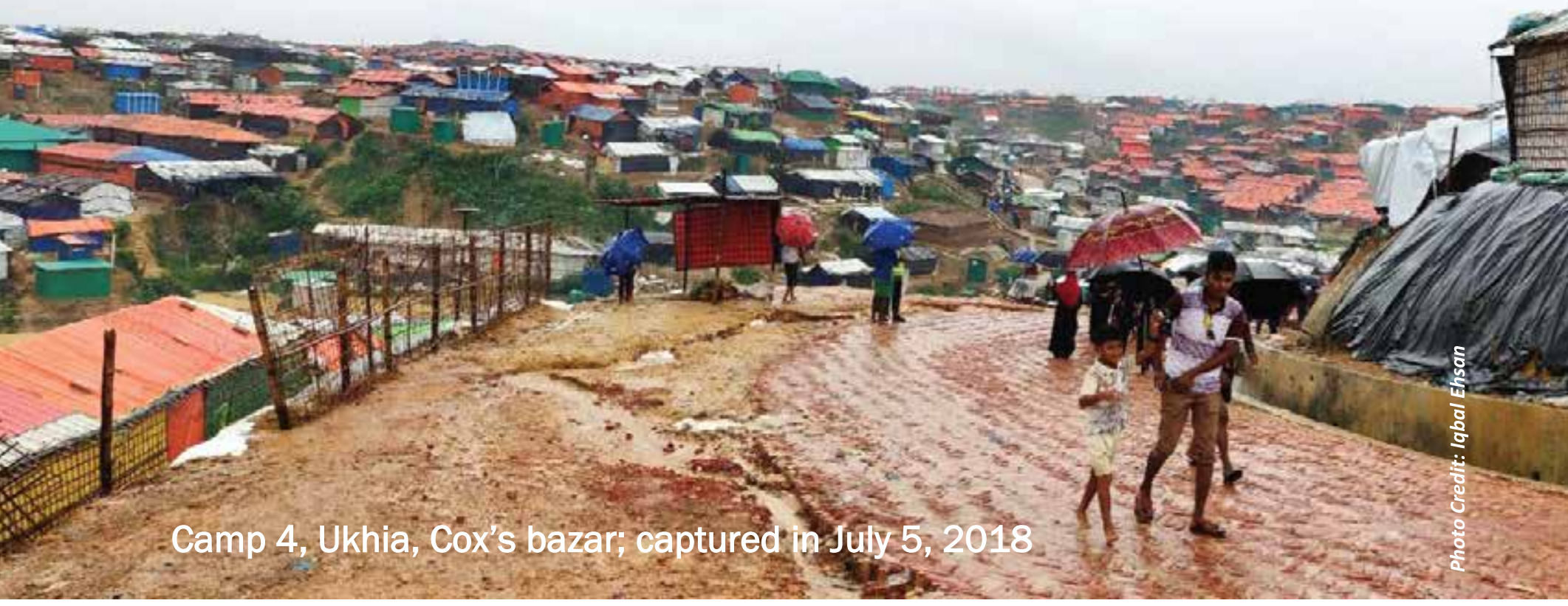

Table 3: Population of data collection sites in Palongkhali Union, Ukhia upazila

\begin{tabular}{lcc}
\hline Camp & Total households & Total population \\
\hline Camp 4 & 6905 & 29009 \\
\hline Camp 8E & 7915 & 33541 \\
\hline Camp 10 & 7943 & 31540 \\
\hline Camp 15 & 10002 & 45130 \\
\hline Camp 20 & 1999 & 8173 \\
\hline
\end{tabular}

The geographical and infrastructural characteristics of all the study sites are similar. A Bangladeshi Governmentappointed representative designated as site management sector coordinator oversees the housing and shelter construction activities in each camp. The temporary shelters are mainly constructed of bamboo and polythene sheets.

\section{RESEARCH TEAM}

The study team was comprised of three Council researchers from the Dhaka office under guidance of two co-principal investigators. Council researchers developed data collection instruments, recruited and trained the data collection team, coordinated and supervised data collection activities, coded, analyzed data, and prepared the report. Seven research assistants (RAs)-four female and three male-were recruited for data collection. RAs graduated from the Faculty of Social Sciences from different public universities and most of them had master's degrees in anthropology. All except one RA were fluent in Chatgaya or the Chittagonian

\section{Ukhia}

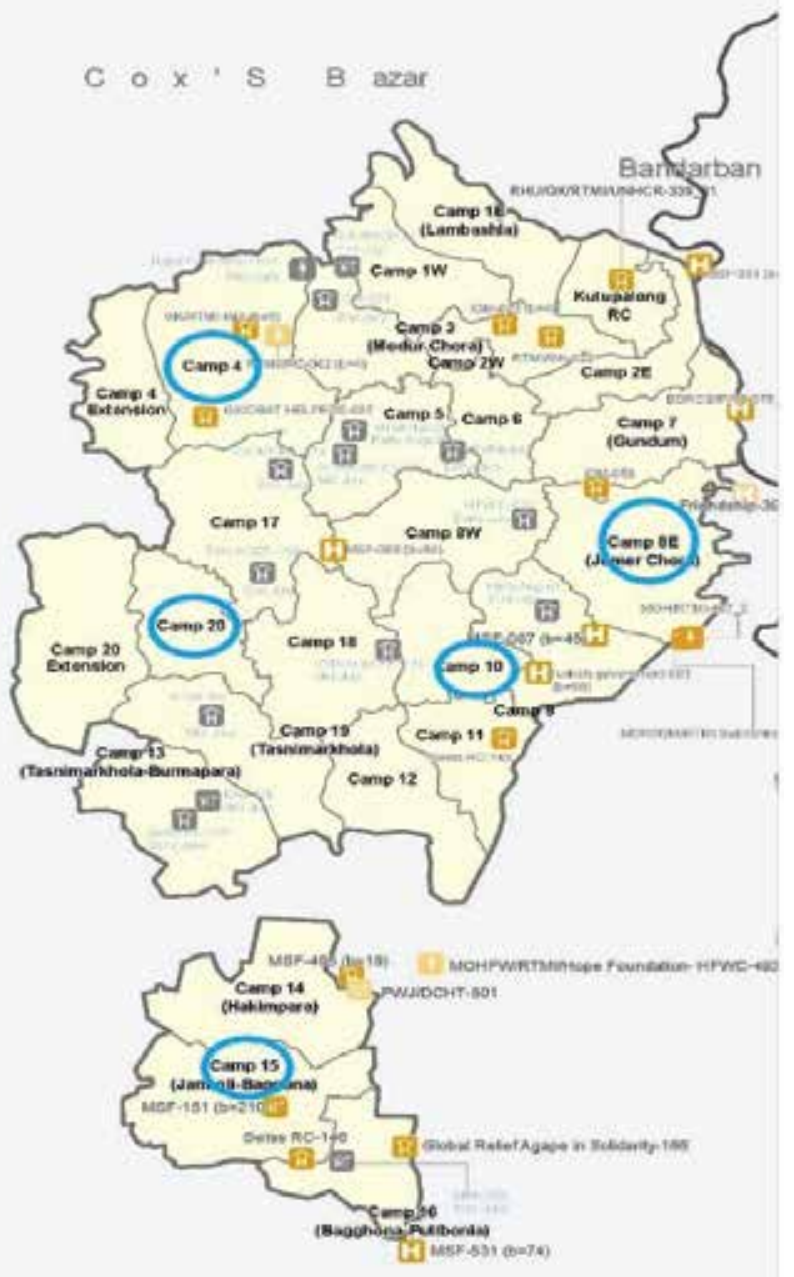

Figure 1: Rohingya Camp location, Ukhia 
dialect which shares 70 percent similarity with the Rakhine language, the difference being primarily related to some specific terminology (Translators without borders 2017). ${ }^{5}$ Three RAs had previous experience working in Rohingya camps and collecting information from Rohingya populations.

RAs received a five-day training in Dhaka on data collection instruments, research methodology, research ethics, and the challenges of data collection in a humanitarian crisis setting. During the training period, the team developed a glossary of important terms in comparable English, Bengali, Rohingya, and Chittagonian dialects. The glossary was continuously updated and modified until field-testing of the instruments. (See annex 1 for glossary).

To avoid confusion and risk of misunderstanding during verbal communication between RAs and Rohingya respondents during data collection process, six Rohingya youths (male and female) from the camps were recruited to assist RAs during data collection in camps. All of these recruited Rohingya youths had some experience in working with their community. The recruited Rohingya youths assisted research assistants in understanding unfamiliar dialects and concepts during IDIs and FGDs and also in identifying eligible respondents through household enumeration.

A two-day-long training was conducted with research assistants and Rohingya youths in Balukhali, Ukhia to teach them the research objectives and methods of data collection.

\section{ELIGIBLE RESPONDENTS}

The study included Rohingya male and female adolescents/ youth, Majhee, Imams and adult women who migrated to Cox's Bazar after August 25, 2017. Data were also collected from service providers and program managers working with the Rohingya population and with adolescents and youth from the host community.

Before data collection, RAs, with the help of Rohingya youths, enumerated 50-60 households in each camp to identify eligible adolescent respondents for in-depth interviews aged between 14 and 24 . After identifying households, the study team approached the household head (when respondent was underage) and/or respondents and followed appropriate consent procedures before conducting the interviews. Majhee, Imams, and adult Rohingya women who live in close proximity to the sample of adolescents were chosen purposively. Brief description of data collection instruments can be found in annex 2 .

\section{RESEARCH ETHICS}

Our protocol and data collection instruments received ethical approval from the Institutional Review Board (IRB) of the Population Council and by the UNFPA. Throughout the study, steps were taken to minimize risks associated with conducting research among young and vulnerable populations. There was a strong emphasis on having young (preferably under the age of 30), same-sex data collectors to generate a sense of comfort and understanding with adolescents. Data collectors were also fluent in the local language which allowed for meaningful engagement with adolescents and youth and the collection of detailed information. Data collectors were attentive to issues that might trigger distress and received training on ethics and child protection. The data collection tools were designed to limit the likelihood of exposing girls to discomfort. Data collectors were aware of referral mechanisms in place for those who experienced distress and required follow-up support.

Protecting and respecting the confidentiality, anonymity, and privacy of respondents was critical and was maintained through data collection and management procedures that ensured that the identity of respondents remained anonymous. All staff involved in this study received appropriate training on research ethics emphasizing the importance of informed consent and confidentiality. At the beginning of data collection, interviewers assured study participants that refusal to participate in the study would not affect

\footnotetext{
5 According to the TWB language assessment held in 2017, Rohingya speakers estimated that there was a 70 percent similarity between Chittagonian and Rohingya (TWB 2017). It also noted that a 90 percent similarity was estimated between the Cox's Bazar dialect of Chittagonian and Rohingya because of the close geographic proximity of these two regions (Mourtada et al. 2017).
} 
their personal or professional life. All interviewees gave their informed consent to being interviewed and to having the interview audiotaped. Research assistants clearly informed the respondents about the purpose of the study and the use of data. Digital recordings of IDIs and FGDs did not contain any identifying information that could link the transcripts to the identities of individual participants. Only the research team were authorized to access any collected data. Pseudonyms are used throughout the report so that responses cannot be traced back to individuals.

\section{DATA ANALYSIS}

Following data collection, the researchers analyzed the data and prepared the research findings and report. It is important to note that this research study is not designed to establish prevalence, or to provide a quantitative or objective account of the experiences of Rohingya adolescents and youth. Consistent with the methodology, the goal of the data analysis has been to draw out and amplify the voices of Rohingya adolescents and youth in the research findings. The findings underscore the fact that they are not a homogenous group and do not have a single view or set of experiences in crisis contexts. The report highlights areas of commonalty and difference, which provide a rich understanding of the experiences and needs of adolescent and youth in crisis.

All IDIs and FGDs were audiotaped and transcribed into Bengali and translated into English. They were reviewed and checked for accuracy and completeness. All data were stored in password-protected computers. Hard copies of the IDI and FGD transcripts were stored securely in a locked cabinet in accordance with Population Council data protection policies.

Triangulation of data sets (IDIs and FGDs) and triangulation from researchers both were part of the analysis. Three senior researchers from the Dhaka office read the same transcripts and coded individually. The codes were then compared and summarized to generate a common code book and themes emerging from data. Both manual coding and a web based qualitative data analysis software, Dedoose, ${ }^{6}$ were used for organizing, coding, and analyzing data.

\section{STUDY LIMITATIONS}

Despite efforts made to include a representative cross-section of adolescent girls in the data collection processes, the sampling approach may have missed some of the most marginalized girls due to the limited visibility of these girls in the community. Consequently, the research findings may not be fully representative of the most marginalized girls, including those with disabilities, those who have been orphaned or travelled to Bangladesh unaccompanied by parents, those too ill to participate, and widows or single mothers with limited time to participate in research activities.

This study is also limited due to the challenges faced in working in multiple languages. Data collection tools were first designed in English, then translated in to Bangla for study field-staff. Data collection was conducted verbally in the Rohingya language. Recordings were later transcribed and analyzed in Bangla, and findings were translated back into English before inclusion in the report. Translation in multiple languages creates opportunities for both questions and responses to lose some nuances or meanings. We addressed this challenge by using data collectors fluent in the Chittagonian language and engaging with Rohingya refugees to create a glossary table for key terms in multiple languages. However, we recognize this is a potential study limitation.

\footnotetext{
${ }^{6}$ Dedoose is relatively new tool in qualitative research and is gaining popularity because of its flexibility as a webbased tool and for its ability to perform synchronous collaborative research from different sites by multiple researchers as well as to work for an individual researcher; it provides a range of mixed-method tools for the handling of text, spreadsheet data, and video. A series of workspaces allows coding, retrievals, and memoing, and a wide range of charts enable quantitative visualization of findings if required. More information can be found at https://www.dedoose.com/
} 


\section{CHAPTER 3: MARRIAGE NORMS AND PRACTICES}

This chapter presents the findings from IDIs and FGDs related to long-standing marriage norms and practices among Rohingya, practices in Myanmar prior to displacement, and after their arrival in refugee camps in Bangladesh.

\section{PRE-DISPLACEMENT MARRIAGE PRACTICES AMONG THE ROHINGYA}

\section{Prevalence of child marriage}

Discussions with respondents suggested a strong preference for child marriages for girls but not for boys. The Rohingya attributed this to religious teachings. Girls are considered eligible for marriage as soon as they reach puberty. Boys are considered eligible for marriage only after they start earning.

"Girls are considered eligible after their menstruation starts and boys are considered eligible for marriage by their parents after they start earning money. Parents save money to build home for their sons to get married. Even if the boy doesn't earn, he eventually gets married. It is Allah's order to parents to get their children married once they become mature." (IDI 5, Married woman, age 19)

"Girls are considered eligible for marriage after menarche. Boys are considered eligible when they are mature enough to understand marital life." (Adult Rohingya woman, FGD participant)

Arranged marriage is reported to be the social norm among the Rohingya. Parents' fears for their daughters' sexual purity and involvement before marriage in an “illicit" relationship, with related implications in terms of reduced marriageability, act as motivations for arranging child marriages for their girls. These concerns are heightened by the onset of puberty when girls begin attracting attention to their marriageability.

“About a year after girls' menarche, boys start to disturb them. Many times girls also get involved in romantic affairs with boys. That's why parents want their daughters to marry off at that age."(IDI 6, Married woman, age 20)

“Let's say there are a few young girls in my house who cannot be married off. Even if they are not involved in any kind of sexual act, people will start to speak ill of them. People try to spread overstated rumors and defame them...so the families impatiently try to marry off their daughters as soon as their menstrual cycle starts. My father took decisions about my marriage because of this. Boys have no risk of getting defamed. So, they are in no hurry to get married early." (IDI 10, Married woman, age 20)

These general concerns about the sexual security of young girls and women appear to be further exacerbated by conditions of violence and insecurity.

"After a girl has had their first period, it is no longer safe for them to stay home. Because of the fear of the military and the police. They can come at any time and rape her or abduct her. Even if this threat did not exist, there is another fear-the fear of eloping. If the girl runs away with any boy, there is nothing the parents can do about it. Hence, before any such thing can happen, they marry off their daughter." (IDI 2, Married woman, age 21)

Marriage age and registration practices were reported to be subject to regulations and enforced by the Myanmar authorities.

"Mainly boys get married at the age of 20 years, 21 years or 22 years. Girls get married at the age of 18. This is the law set by the government. Although people do get married 
earlier. Marriages take place at the age of 16 or 17. Girls are married off one or two years after their first period (menstrual cycle)." (IDI 37, Unmarried boy, age 17)

Some respondents described how age restrictions at marriage were violated either by not registering the marriages or through monetary negotiations with the authority, as mentioned in many of the in-depth interviews and FGDs by both married and unmarried Rohingya respondents. Rohingya respondents described bribing military and local government officials to obtain permission and register underage marriages by falsifying age to meet the minimum age limit as per marriage law.

"In our society, girls are even married off at 12-14 years of age, if sufficient amount of money can be spent. Boys get married at about 16-18 years of age. People get married as soon as they are mature enough, and all of this is possible only because of money. If 15 lac Kyat is offered, even a 12 years old girl can also be married off." (IDI 1, Married women, age 20)

"A lot of money was spent to register the marriage. As I was 17 years old, we had to bribe our community leaders. We had to wait for 10 days after paying 3.5 lac in the army camp to get our name registered. All these things were done as getting married under 18 years age was not permitted there. If we didn't bribe with a lot of money, my name could not be registered." (IDI 5, Married woman, age 19)

Relatively wealthier people could arrange early marriages for their daughters more easily than people who were not able to pay the bribe for underage marriages along with the marriage registration fees. The amount of bribe tends to range from 5 lac to 10 lac Burmese Kyat (350-650 USD).

"Usually for boys it is 20 years and for girls it is 18. Those who are not financially solvent cannot marry off their daughters at 18 or even after that; it becomes very difficult for them. For those who are solvent, they can marry off their daughters even before 18 by bribing the social elite and the military." (IDI 4, Married woman, age 23)

\section{Marriage ceremony and registration}

Rohingya families meet and agree upon marriage, the amount of gold and Mahr ${ }^{7}$ is decided mutually, and papers are prepared accordingly, also mentioning the amount to be paid as alimony. A religious leader (Hujur) conducts the marriage ceremony with community members as witnesses, and a Muslim marriage contract is accepted. A ceremonial feast is then arranged for relatives and guests. Finally, the bride is sent to the groom's family along with dowry.

Dowry payments are common and socially accepted among the Rohingya in a manner similar to other South-Asian communities. Demand and payment of dowry is reported as customary both in Myanmar and in camp settings. Respondents mentioned that both in Myanmar and in camps, poor families were unable to give their daughters in marriage because they were not able to pay the dowry money demanded by the groom's family. No marriages are organized without dowry. Everyone pays some dowry (in cash or kind, or both) but amounts vary depending on financial ability.

"The groom's family usually demands money. However, some demand smartphones as dowry. Usually the amount of dowry is equivalent of the amount of gold given to the bride as Mahr." (IDI 16, Unmarried girl, age 15)

“During weddings in our society, the brides' families had to pay the grooms' a lot of dowry, as much as they could afford. The grooms were given cash money, dresses, furniture, cows, properties, etc." (IDI 22, Unmarried girl, age 14)

Reflections on marriages prior to migration to Bangladesh suggest that there were strong preferences for child marriage and these existed despite state control to curb the practice and regulate it. Respondents described a consistent pattern of control and oversight by local Myanmar military administrators to implement Myanmar law, which specifies the minimum age of 18 for girls and 20 for boys.

\footnotetext{
${ }^{7}$ Mahr is a mandatory payment or possessions given by the groom to the bride after the solemnization ceremony that legally become the bride's property. It symbolizes the beginning of a husband's responsibility towards his wife. Mahr can be in cash or kind, such as gold or silver.
} 
“In Myanmar you cannot get married without a Lathung (marriage permission letter that Rohingya had to get from the military prior to their marriage). If you did get married without it, they would beat you, fine you and chase you. Lathung is a kind of agreement and the law dictates that you cannot get married without it." (IDI 26, Married man, age 19)

Respondents consistently reported that not registering a marriage with the authorities is a punishable offence and described their experiences.

"Money is required at the time of marriage, about 1 lac kyat; then, at the time of preparing the papers and documents, you need 1 to 2 lacs again. You are not allowed to marry before the age of 18. And if anyone marries in secret and the government finds about it, they beat them up and fine them heavily. Getting married there (Myanmar) is a very difficult matter." (IDI 29, Married man, age 21)

\section{MARRIAGE PRACTICES AND PATTERNS AFTER DISPLACEMENT}

Qualitative study findings captured some significant changes in marriage patterns and practices after displacement. Unlike in Myanmar, there is no age restriction for marriage in the camps. Hence, Rohingya girls and boys are getting married as early as 14/15 years of age. Marriages are arranged based on mutual agreement between the two families, and the camp Majhee is usually informed. The amount of gold that the bride receives from the groom and the dowry amount received by the groom from the bride's family are written on a white paper and signed by both parties.

"Here (in camp) marriages can be performed easily. There is no restriction on age for marriages. Marriages can be performed at an early age... There is no rule of law relating to marriage in camp. People can marry as they wish... No registration is required here (in the camp) as it was in Myanmar." (Adult Rohingya woman, FGD participant)

These sentiments were echoed by others, indicating that marriage transactions have become less formal and rigid compared to how they were reported to be in Myanmar but the demands for dowry have increased:

"Here (in the camp), there is no bar on marriageable age. You need to inform Majhees before arranging a marriage. Here the groom's family demands dowry. People are not financially solvent enough here. Hence dowry is paid according to families' capability. But demand of dowry is increasing day by day leaving a number of girls unmarried." (IDI 17, Unmarried girl, age 15)

“In camp we don't have to pay anything to Mogh army. However, the bride's family has to pay dowry to groom as per their ability. No marriage can be arranged without dowry. Hence, girls of poor families remain unmarried but girls of wealthy families are getting married." (IDI 12, Married woman, age 23)

\section{Marriage ceremony in the camp}

Arranged marriages are still the norm among displaced Rohingya living in refugee camps; however, financial constraints have forced changes in traditional practices. Respondents said that goods and services are more expensive in camp than in Myanmar, and as a result, weddings in camps tend to be less festive and ceremonial.

"I wish we could celebrate the weddings here with the same festivity, have fun, and slaughter cows to feed guests, like we used to do in Myanmar. Several kilograms of beef are purchased here to feed guests. Weddings are celebrated here in this way. None of the customs of our country are being followed here due to financial constraint." (IDI 8, Married woman, age 20)

"There is scarcity of everything here. Weddings are arranged in a minimal way here. The groom's family comes to see the bride. If they like the bride, they confirm the amount to be paid as Mahr and fix the marriage. Afterwards, they take the bride walking to their home." (IDI 6, Married woman, age 19) 
Many respondents mentioned that financial insecurity impedes marriages of girls in the refugee camps. Families are unable to arrange marriages for their children because they don't have enough money to pay dowry or to conduct marriage ceremonies. Respondents mentioned that Rohingya who have relatives living abroad and receive remittances regularly are able to arrange marriages for their children.

"Those who can afford it can marry off their daughters; poor people cannot marry off their daughters. Those who have relatives living abroad to earn money and send money for them from abroad, they marry off their children with the money and gold." (Adult Rohingya woman, FGD participant)

There is some evidence that marriage preferences are also dictated by the place of origin of the bride and groom.

"People want to fix a marriage with families who used to live in the locality or are from the same community as them in Myanmar. If not available, then people arrange marriages outside of their own community." (Adult Rohingya woman, FGD participant)

\section{Not enough eligible groom compared to marriageable girls}

Respondents reported there is a perceived deficit of men, and conflict and displacement have led to an imbalance of the sex ratio in the camps, which has had an impact on marriage practices.

"The number of girls is more compared to that of boys here. Because the men are going abroad for work, but the girls have been left at home." (IDI 8, Married woman, age 22)

"Number of marriageable girls has increased [more] than number of eligible grooms in Rohingya community. Many men have been killed in Myanmar. Many men are living in Malaysia or in other foreign countries. As a result, in every house there are 3, 4, or 5 unmarried girls. In my father's house there are 3 unmarried girls." (IDI 1, Married woman, age 20)

When asked whether the deficit of men meant that Rohingya women are marrying Bangladeshi men, respondents asserted their regional preferences and said that a Rohingya only marries another Rohingya and they do not marry outside of their community. As we report in Chapter 7, the host community respondents stated conversely that Bangladeshi men are marrying Rohingya girls in the camps. 


\section{CHAPTER 4: FAMILY PLANNING AND CHILDBEARING NORMS AND PRACTICES}

The Rohingya population has been historically excluded from large scale enumeration and national surveys in Myanmar due to the interethnic political crisis. Thus, the national Total Fertility Rate of Myanmar (2.3) is not necessarily representative of the Rohingya population. The recent icddr,b study provides some estimates of their family size, contraceptive use, and choice of FP methods, but the reasons for low usage of contraceptives and limited service uptake are still unknown.

This chapter aims to explain the factors that influence the sexual and reproductive health behaviors and practices of the Rohingya living in the camps, and thus complements the icddr,b study estimates and further explores the possible gateways to intervene in belief systems and practices of the Rohingya with effective programmatic actions. Data from IDIs and FGDs with Rohingya adolescents and youth and IDIs with service providers and program managers form the basis of this analysis. Findings reveal the underlying social, cultural, and historical context that shape Rohingya perspectives on childbearing, family planning, and contraception. We also assess the changes on the acceptance of services, including family planning methods, experiences in Myanmar prior to displacement compared to after displacement living in the camps in Bangladesh.

\section{PREFERENCE FOR LARGE FAMILIES}

The most common reason for a preference for large families among the Rohingya is that it has been the practice of past generations. Respondents reported that their parents and grandparents were also from large families. Their childbearing norms and practices indicate that limiting the number of children by using contraception is not prevalent among the Rohingya in Rakhine state. Women are encouraged to have as many children as they can.

\begin{abstract}
"My mother gave birth to 11 children including me. Her father, my grandfather, had two wives, and he had a total of 17 children including my mother... I don't know how many children I would give birth to in my lifetime. It depends on the will of Allah" (IDI 12, Married girl, age 17).
\end{abstract}

Box 2: Desired family size
Rahima (pseudonym) is a 20-year-old married
girl living in camp 8E. She came to Cox's
Bazar, Bangladesh in August 31, 2017 with
her husband and two children from Myanmar.
During the time of data collection in July
2018 , the age of her younger child was 14
months and her elder child was 30 months
old. She was married at the age of 16 and
delivered her first child at the age of 17.
Rahima reported that she is currently living
with her husband and not using any
contraceptive method. Rahima's mother had
6 children and her mother-in-law had 5
children. Her intention is to have as many as
children as she can, and she is not interested
in using a contraceptive method in future. (IDI
3, Married girl, age 20)

\section{Dominant religious beliefs}

In addition to the generally pronatalist and fatalistic sentiments described above, the belief that Islam prohibits the use of contraceptive methods was commonly reported among the Rohingya. Respondents mentioned that having more children is encouraged by Islam.

"The Rohingya feel that more children bring prosperity and happiness in the family because they are the gift of Allah. Humans cannot create children by themselves. So they should conceive when it comes normally... the Huzurs (Muslim religious leaders) tell us not to use a contraceptive method. Contraception is a sin. Allah made women fertile 
so that they can bear children. So why would we create a barrier? We feel blessed when a child is born in the family. So why would you disobey Allah?" (IDI 2, Married woman, age 21)

"Using contraceptive methods for limiting children is a sin in Islam. Allah gives us children, we should not stand against the will of Allah. He will feed them, he will raise them. It is not good to use injection for avoiding pregnancy... We do not use any contraceptive method. We will take as many children as Allah gives." (IDI 34, Married man, age 23)

Religious leaders, mainly Imam, play a crucial role in spreading religious beliefs in the society. During the FGDs with Imam, they mentioned that Islam does not permit the use of contraceptive methods for limiting pregnancy.

"In the Quran, it is clearly said that children are the gift of Allah. Some women are taking medicine to avoid pregnancy which is a sin (haram) in Islam. It is a great sin. God will definitely punish them for that crime, and the couple who use them will have to give explanation on the Day of Judgment for taking contraceptives. Many women also take medicine for abortion, and this is also a great sin in Islam." (Imam, FGD participant)

Although the use of contraception is a sin according to their Islamic beliefs, there is evidence that some respondents practice contraception, and it can be acceptable on the basis of health.

"My wife took the oral pill while we lived in Myanmar. She took one pill each day. We wanted to avoid having more children because she was not physically well." (Imam, FGD participant)

"I think after having a baby, it is a good idea to wait at least two years before getting pregnant again. It is good for the mother's health. Moreover, the mother can take care her newborn baby properly." (IDI 28, Married man, age 21)

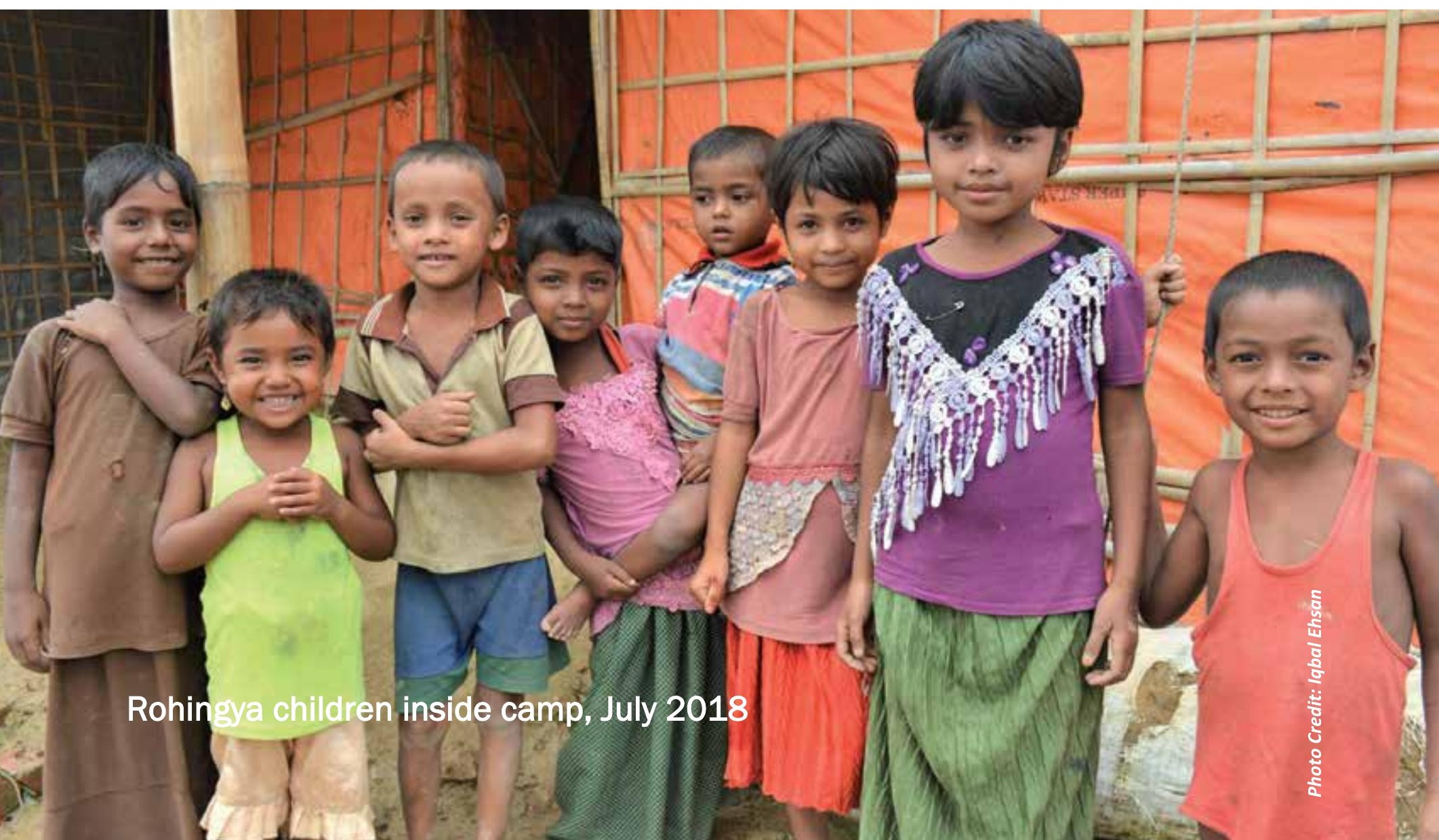




\section{Societal norms and influence of gatekeepers}

Among the Rohingya, there are strong societal and family expectations for women to become pregnant and give birth soon after marriage. Family members, especially husbands and mothers-in-law, play a crucial role in decisions related to the child bearing and contraceptive choice of a married girl.

"I became pregnant in just two months after my wedding. My husband prohibited me from using any contraceptive method to avoid pregnancy. He also warned that if I start taking the contraceptive pill just after my marriage, I would not be able to conceive a child in the future. And my husband also believes that there is no risk in having as many children as possible... Just like my husband, the rest of the community also see it in a positive way. Young girls think that their age is perfect for conceiving a child. That is why they do not use contraceptive methods." (IDI 4, Married woman, age 23)

"My family members view contraceptive methods negatively. Nobody would want to adopt these methods. I've heard little about these methods in Myanmar. I've never adopted these methods myself. My family members didn't approve it. I haven't used these even after coming to this camp." (IDI 13, Married woman, age 20)

"My parents forbade me to adopt these methods. They said these are prohibited according to Islamic Shariah ... Adopting birth control methods is discouraged in our society because it is believed to be prohibited in our religion; it causes illness, and it makes menstruations irregular." (IDI 9, Married woman, age 22)

A program manager explained their perception of the decision-making process of contraception.

"In many Rohingya families, even if the wives are interested in family planning services, their husbands or in-laws restrict them. However, those who are conscious take these services secretly. For this reason, it is necessary to involve the husbands and mothers-inlaw to make them understand these issues. We need people to provide communitybased services for raising awareness." (Program personnel, NGO)

\section{Stigma and misconceptions about contraception}

Deep-rooted stigma and misconceptions about contraceptive methods exist among the Rohingya. Respondents reported that in Myanmar contraceptive methods were accessible, but most of the Rohingya were reluctant to use contraception because of fears of permanent sterility and other morbidities.

"Most of the people believe that if anyone uses it once, they will not be able to conceive anymore in the future. The contraceptive user will lose her fertility. They also believe that contraceptive methods will cause irregular menstruation and prolonged menstrual bleeding. Later, for treatment of these side effects, women will have to visit the doctor, and incur extra expenses for the family. So they do not use it." (IDI 3, Married woman, age 20)

Even unmarried girls who have not had any exposure to sexual or married life reported having misconceptions about the medical consequences of contraceptive methods.

"We are afraid that menstrual cycles will become irregular, or women might get infections in the uterus for adopting birth control methods." (IDI 20, Unmarried girl, age 17)

Interviews with service providers suggest that a preference for large family size is the primary hurdle in the promotion of family planning.

"One of the main obstacles is that they have a lot of children. Many women who already have a lot of children are conceiving again. If they are advised to use FP method or do $M R$ [menstrual regulation] for unintentional pregnancy, they say, 'Why would I do this? 
As Allah has given [the child], it should be born. Aborting it will be a grave sin.' They don't want to adopt a family planning method. They say that if they do, they will become sick and infertile." (Service provider, paramedic)

One way in which norms of low contraceptive use are perpetuated is by associating contraception use with immorality and illegitimate sexual relationships.

"Only those who are sexually active in secret need to use condoms and other contraceptives ... People like us who are not involved in sexual relations don't need to use condom. But those who are sexually active before marriage need to use a condom, so they can pretend to be virgin ..." (IDI 14, Unmarried girl, age 14).

\section{Fear of side effects of contraceptive methods on women's health}

Even for women who reported that they are willing to try family planning methods, the general environment of skepticism and fear, particularly about health side effects, can motivate discontinuation. Findings suggest that the Rohingya fear the side effects of contraceptive methods.

“I stopped using injections because I couldn't adjust to them. It has side effects on my health; I used to experience lightheadedness and weight loss. I used to feel weak. So to stay fit, I had to take vitamin supplements along with taking injections." (IDI 7, Married woman, age 22)

Experiences of side effects by women from the community influence others in decision making process of contraceptive choices.

“Many women feel dizzy after taking birth spacing pills. They become bedridden. Various types of illnesses appear, like gout, body ache, etc. More vitamin supplements have to be taken during this time. I've heard about many cases where men divorce their wives because they become sick after taking these medicines." (Adult Rohingya woman, FGD participant)

"I have heard that women suffer from many adverse side effects like headache, lightheadedness, irregular menstrual cycles, and heavy prolonged bleeding. Their body can't adapt to these methods." (IDI 22, Unmarried girl, age 14)

\section{CHANGING ATTITUDE TOWARDS CONTRACEPTION IN CAMP SETTING}

Despite traditional religious beliefs against contraception and fear of their side effects, awareness of the benefits of using contraception seems to be growing among younger generation living in the camps.

"I've heard about birth spacing pills and depo injection from my sister-in-law. We call it 'Card-Dabai' (oral pills). A single card is taken per month. Each card has 30 tablets. One tablet has to be taken each day. If a day is missed, then the card has to be discarded and a new card has to be started." (IDI 14, Unmarried girl, age 18)

"People from the previous generation have negative perceptions towards contraception. They take it as a sin. But now, most people think that it is a good thing. Many say that if you can't feed them properly or take care of them, then what is the point of having so many children?" (Adult Rohingya woman, FGD participant)

"I am still young, I am not thinking about taking any such measures now. However, I think it is better to follow some birth control method after having 4-5 children, for the benefit of the mother." (IDI 9, Married woman, age 19) 


\section{Box 3: Future intention of family planning}

Sadia (pseudonym) was married at the age of 14 and gave birth to her first child when she was 15. She came to Cox's Bazar, Bangladesh in November 2017 with her husband and son. At the time of the interview in July 2018, her son was 16 months old and she was pregnant again. She has 11 siblings. She is not currently using any contraceptives, but is interested in using injectable contraception after childbirth. She feels it is not suitable for her to have more children in the current unstable situation in camp. She learnt about the injectable method from a health worker in the camp. (IDI 13, Married girl, age 17)

FGDs with Majhees indicate that the use of contraceptive methods has increased in the camp compared to the time in Myanmar.

"When we were in Burma, we had very few doctors. Hospitals were far away; if we had any major illness, we had to spend a lot of money for doctors. We didn't receive such services or didn't know about these due to the lack of doctors. That is why the number of children among the Rohingya has increased so much. In Bangladesh, NGOs and the government have established many hospitals; now we are learning about these things. Our women are getting to know about family planning. If they follow family planning, their sufferings will decrease because they will not have children so frequently."

(Majhee, FGD participant)

In the same FGD, another Majhee made the following statement, with which most participants agreed.

"After coming to Bangladesh, those who are learning the difficulties of having many children are consulting with the service providers of different organizations and are now using contraceptive methods." (Majhee, FGD participant)

The changing perception about contraception has also been observed by service providers working in the camps who feel that the supply of services is in part helping to generate demand.

"They have very little idea about SRH and contraceptive methods. They are gradually coming to know about these from us. Now they regularly come to receive these services. They return by themselves for further services after completing their courses. They have become more aware now. Previously, they didn't have knowledge about these issues, but now they do." (Service provider, Paramedics)

\section{INADEQUATE KNOWLEDGE ABOUT HIV/AIDS AND STI}

Findings indicate that the Rohingya have inadequate knowledge about HIV/AIDS and STIs. Some reported that they have heard HIV/AIDS mentioned in Myanmar by the NGOs. Many respondents mentioned that they learned about these health concerns after arriving in Bangladesh.

"I have heard about it in Myanmar. If any boy has sexual relations with several girls then the boy can suffer from HIV/AIDS ... If anyone takes food in the same plate used by an HIV infected person, the disease will spread to the uninfected body." (IDI 19, Unmarried girl, age 16) 


\section{CHAPTER 5: SERVICE SEEKING BEHAVIOR AND PRACTICES}

Rakhine is the least developed area of Myanmar, and access to healthcare across Rakhine State is generally very poor. The Rohingya have been excluded from access to facility-based basic health services for decades in Myanmar, therefore, NGOs and traditional healers are the major healthcare providers in Rakhine State.

This chapter describes health-seeking behaviors and practices of the Rohingya related to SRH, MR, and maternal health services, as well as STIS, HIV, and AIDS, and utilization of the health services available in the camps. Data triangulation from FGDs and IDIs with the Rohingya and FGDs with service providers and program personnel have been used to capture the multidimensional aspects of their health-seeking behavior.

\section{DEPRIVATION FROM MATERNAL HEALTH SERVICES IN MYANMAR}

Findings indicate that service seeking behaviors and practices of the Rohingya are shaped by the decadeslong systematic deprivation process that they were subjected to in their homeland. During IDIs and FGDs, respondents stated that they were not allowed to go to the hospitals due to their restricted mobility. They did not know how to access services from health clinics or hospitals. They were mostly dependent on local pharmacists and traditional healers.

"No healthcare facility was available there (Myanmar). For emergency health treatment, we used to come to Bangladesh. The hospitals in Sittwe were only for the Burmese people, we were not allowed to enter any Burmese hospitals ... I didn't receive any maternal health services in Myanmar. The violence was ongoing during my pregnancy. How could I have been possibly consulting a doctor under so much tension?" (IDI 1 , Married woman, age 20)

“There weren't many government hospitals. The one was in Sittwe town. The people living in the town could go there. Those who lived in the hilly region and remote villages were not allowed to visit the town for availing better health services. There were strict restrictions on our movements. So, we went mostly to private doctors who used to provide services at pharmacies. These doctors charged a lot." (Adult Rohingya woman, FGD participant)

\section{SERVICE COVERAGE IN THE CAMPS}

In order to understand the availability, quality, accessibility, and other challenges related to SRH, FP, and maternal health services for adolescents and youth, information was collected from program personnel and service providers who work in different government departments and non-governmental organizations in the camps. Most of them reported that the health sector is actively providing services in the camps.

"The Bangladesh Government, with the assistance of development partners, has managed the situation effectively. We have ensured 100 percent vaccination among the children. We have been maintaining a strong network with the implementing partners and donors to ensure proper monitoring. We have prevented diseases like diarrhea and diphtheria successfully. We have established sustainable referral linkage to hospitals through basic health units. At present, the contraceptive prevalence rate is more than 
30 percent, up from almost zero when they were in Myanmar. Many Rohingya women now visit the facilities in the camps for ANC and PNC services. However, we have a shortage of funds. The health sector needs additional funds for ensuring a better healthy life for the Rohingya." (Civil Surgeon, Cox's Bazar)

National and international NGOs have been providing healthcare services in the camps in different modalities to ensure effective outreach among the Rohingya.

"Two types of services are available here from our organization. One is facility-based health services and the other is outreach. We provide counseling on SRH. We cover different components of SRH, such as menstrual management, GBV, family planning, etc. We provide counseling for family planning-both short-term and long-term methods. Among short term methods, birth spacing pills, condoms, and Depo-Provera injections are available in our facilities." (Program personnel, SRH-focused INGO)

\section{SRH and other common health problems}

Interviews with Service Providers revealed that married adolescent girls are experiencing early pregnancy, low birth-weight for newborns, and anemia and malnutrition related health problems. Unmarried girls generally suffer from scabies, leucorrhea, irregular menstruation, anemia, and malnutrition. Diarrhea, jaundice, weakness, fever, cold, cough, and skin disease are common health problems for the adolescents and youth. Adolescent boys hardly visit health facilities; this is identified as a service delivery gap by some service providers and program managers.

"It is not possible for us to reach everyone. Unmarried girls are not allowed to go out of their house. In this case, they are being left out. Family members believe girls should not go out of their house after they become 10-12 years old. Girls of mainly this age are being left out of our service. Unmarried boys are also not coming for SRH services." (Program Personnel, National NGO)

\section{Satisfaction with maternal health services}

The Government of Bangladesh and national and international NGOs have been providing essential maternal health services in the camps. Many respondents said that they had not received such high-quality health-care services in Myanmar.

"I've heard that there are many facilities for mothers and babies in the camp. They provide free medicine and vaccine to the mother and the newborn. They even provide new clothes, baby food, and other goods for newborn babies. We never received such things in Myanmar." (IDI 3, Married woman, age 20)

Male adolescents, too, acknowledged and appreciated the availability of healthcare services for pregnant women in the camps.

“Services are available in the camp for pregnant mothers. Service providers and field workers help pregnant women in many ways. They behave well with everyone. They provide healthcare guidance to mothers. They provide services in emergency situations. They also provide information on how to purify water with medicine before drinking or cooking; they advise them to wear sandals before using the toilet and to keep the toilets clean. They also provide free medicines for pregnant mothers in the hospital." (IDI 25, Married man, age 25) 


\section{Home birth is a common practice}

Respondents reported that home birth is a common practice among the Rohingya. Historically, barriers to accessing facility-based maternal health services pushed the Rohingya to manage childbirth at home using traditional birth-attendants.

"The Rohingya prefer giving birth at home. In Myanmar no one went to hospital to give birth, but here in Bangladesh people go to hospital to give birth. We heard about facilitybased maternal services only after coming here. In Myanmar, everyone delivered at home with help of a 'Dhoroni' (local birth-attendant). We did not even know about delivering in the hands of a doctor. We could not have afforded it. It took around 8-10 lac Kyat (500-600USD) to go to the hospital to deliver baby. That is why, due to lack of money, everyone gives birth at home." (Adult Rohingya woman, FGD participant)

"My first child was born at home. I suffered a lot but still did not go to a doctor. If one goes to hospitals and gives birth to a boy child, they (Mogh) kill the baby. In this fear, I didn't go to the hospital ... It is better to suffer at home instead." (IDI 6, Married woman, age 24,)

Respondents expressed fear and had misconceptions about facility-based delivery. Some believe that if they visit a hospital during their delivery, doctors will forcefully impose a C-section.

"Women were afraid to visit health facilities for delivery in Myanmar. I've seen this fear among many pregnant women in the camp too. Our volunteers tried to convince them to visit a hospital during delivery, but they thought if they visit the hospital, the doctor will cut their belly. They have extreme fear about C-section. We are counseling them to reduce the fear." (Service provider, Medical Officer)

The icddr,b study findings corroborate this fear; it is estimated that 54 percent of pregnant women had not decided about the place to deliver their baby. Only 10 percent pregnant women expressed their wishes to deliver at a facility, and the remaining 36 percent opted for home (Chowdhury et al. 2018).

\section{Service uptake in health facilities and associated barriers}

We explored challenges to the accessibility of health services as well as perceived barriers that prevent women in the camps from seeking services. Almost all respondents reported that health services are available for children, pregnant women, and adult populations. Although they are aware of the existence of healthcare services, utilization is very low.

"A majority of the patients, most of whom are women, are married. We receive few male patients. Generally, men do not come for SRH services because they are not aware. We are promoting condoms in our program, but the response is very low." (Program Personal, SRH-focused INGO)

"They think condom or any contraceptive method is prohibited in Islam. So, they do not use any contraceptive method. Once, I asked a married Rohingya man who visited our facility for syphilis the reason behind it [non-use]. After treatment, we advised him to use condoms. He replied that 'Rohingya men do not use condom. It is prohibited in Islam.' We have found this perception among many men in the camp." (Program personnel, SRH-focused National NGO)

Service providers identified lack of awareness as the main reason for not visiting health facilities despite the presence of a large number of facilities in the camps. Transportation and the restricted mobility of adolescent girls were also identified as barriers. 
"The transportation facility available here is not good. The camp area is huge and the condition of the road is very bad. For this reason, adolescents face problems in coming here. Besides, there is also a psychological barrier. Though the adolescent girls want to receive treatment, they don't want to come out of their house. The Rohingya don't allow adolescent girls to go out of their houses." (Service provider, Medical Officer)

"A few days ago, a young woman in the camp was suspected of having an abortion. She was experiencing bleeding. She couldn't come to the healthcare center. So we sent a midwife to deal with the case, and she found that the woman had an incomplete abortion. We referred her to the Turkish Field Hospital for better treatment. We couldn't find out how it had happened. But I guessed it must have happened after taking MR medicines." (Service provider, Medical Officer)

A few program personnel reported that outreach programs were becoming more effective at reaching these populations.

"At the beginning in October 2017, they used to come to receive general healthcare services like treatments for fever, burn injuries, cuts, tears, wounds, diarrhea, etc. None of them used to come for receiving services related to family planning, STD, or ANC and PNC services, safe delivery, GBV, etc. After our continuous effort, it has been seen that about 20 percent of the patients coming to us are adolescents specifically seeking SRH services. This became possible after initiating outreach programs. We observed that adolescents had some demands and barriers for which we started our outreach program. For community engagement, we have appointed volunteers from the Rohingya and also from native Bengalis. Volunteers from both communities are working in pairs. As a result, now we've gained easy access in the community." (Program Personnel, SRHfocused INGO)

Some program personnel also reported that they counseled Rohingya women to use contraception using Islamic scripture to support such use, which they found to be an effective strategy.

“At the beginning, they weren't interested in taking contraceptive methods because of their religious beliefs. Now, after they are being counseled by citing references from the Quran and the Hadith, they are interested in adopting these methods. The Rohingya believe that Allah sends babies to parents, so it should not be prevented. We give them examples from religious scriptures that a mother needs to breastfeed her newborn children up to 30 months for proper growth. If people keep on having children every year, they can't properly feed their children. After hearing such references from the Quran and the Hadith, they are becoming interested in adopting contraceptive methods." (Program personnel, SRH-focused INGO) 


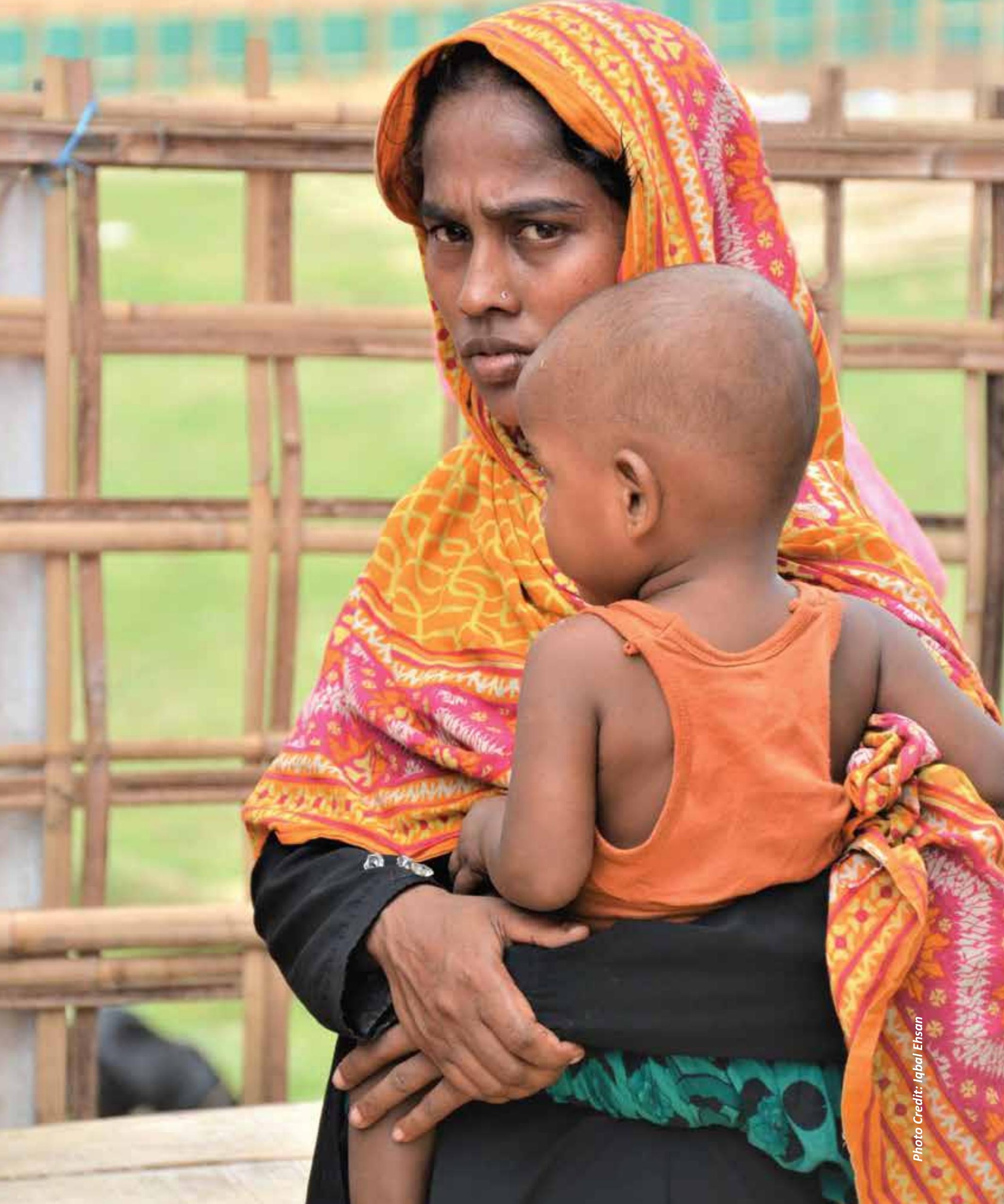




\title{
CHAPTER 6: SAFETY AND SECURITY
}

\author{
Thousands of Rohingya made perilous journeys from Myanmar to Bangladesh to escape communal \\ violence and persecution which was described by the United Nations as a "textbook example of ethnic \\ cleansing." Their experiences in Myanmar continue to have a deep impact on their sense of safety and \\ security even after arrival in Bangladesh (Oxfam 2018).
}

This chapter describes the violence and struggle experienced by the Rohingya population during and after their migration to Bangladesh. The findings are related to safety and security concerns among the Rohingya in the camps and their involvement in risky behaviors such as substance abuse and forced and transactional sex.

\section{EXPERIENCE OF VIOLENCE}

In order to understand the Rohingya's trauma and their experience in the camps, we inquired about their experience while traveling to Bangladesh. Many respondents narrated tragic stories and the extreme hardships faced while crossing the border into Bangladesh. On their way, they witnessed burned villages, dead bodies, and extremely violent acts. Some reported experiencing severe starvation and diseases like diarrhea during their travels.

\begin{abstract}
"Our houses were set on fire using rocket launchers, and burned down to ashes. People were slaughtered and shot or burned to death. Girls were abducted and raped. The girls who had been abducted could not be rescued. They were either killed or kept in barracks. That is why we have fled here." (IDI 15, Unmarried girl, age 17)
\end{abstract}

\begin{abstract}
"Before I came here, the Mogh took my mother on a boat and set it on fire with petrol. They were torturing us a lot. They burned our entire block, harassed our mothers and sisters, and did not let us perform our religious rituals. They tortured us in every way they could-slaughtered us, stabbed us with knifes, threw little children in the fire, burnt old people, and shot many." (IDI 25, Married man, age 24)
\end{abstract}

\section{Box 4: Violence and the fight out of Myanmar}

Amina (pseudonym), age 20, shared the story of her ordeal:

"We had suffered a lot, that's why we came here. We never anticipated that the situation would deteriorate so much on that evening. We didn't even have any idea that something dangerous was going on inside the military base. It was the first night of the month of Dhul-Hijjah. At about 2 am, past midnight, suddenly our houses were set on fire, people were shot down, and no one could understand the situation at first. People were fleeing like insects. One of my aunts along with her three children and one of my sisters along with her three children together hid inside a bathroom. But when the soldiers saw them, they shot my relatives. They died on the spot. When my sister went to save my aunt, she was also killed. A girl in our village was shot in her thigh. The soldiers thought that she was dead and left her behind without checking. When the soldiers went away, the people of our village took her behind a nearby hill. We stayed at that hill for about 15 days. We came out from the hiding place when we couldn't bear the hunger anymore. We moved from one village to another, ate whatever we could find, and cooked burnt rice. Despite having diarrhea, we kept eating that food. We couldn't get medicines as every store, including pharmacies, was burnt down. We drank dirty water from canals and suffered from more diarrhea. One night, when we couldn't bear it anymore and came down from the hill, we heard that the Prime Minister of Bangladesh, Sheikh Hasina, had opened refugee camps to give us shelter. Hearing this, we walked for 3 days by the river bank without food. After 3 days, we reached an estuary, where the boats used to anchor. We found everyone waiting there. Boys were swimming there. People were waiting for the low tide. Everyone was crying. Adults who could be easily transported were being sent away by boats. The elder people were waiting. We left that place and reached another estuary. Here people who could pay money were transported to the other side of the river. Those who didn't have money were crying for help. We saw some boats were leaving and tried to board them. But people started fleeing when they saw the Burmese soldiers coming. Some of us took the courage to bribe them with gold and whatever valuables we had and managed to escape. Those who couldn't board the boats were killed. I've witnessed all this mayhem with my own eyes. We came here after a lot of suffering." 


\section{SECURITY CONCERNS IN THE CAMPS}

Respondents reported that compared to their condition in Myanmar, living in camp is much safer for them. Despite the fact that living in camps can be stressful in many ways, with cramped accommodation in makeshift shelter homes, inadequate water and sanitation facilities, limited access to resources, and restricted mobility within demarcated areas, the Rohingya respondents still feel that camp life is much safer than their villages in Myanmar. Almost all respondents said they don't have any fear, nor do they feel insecure in camp.

"In Myanmar we used to hear gunshots every day. We were living in constant fear. Here we don't have any such fear inside the camp." (IDI 19, Unmarried woman, age 19)

\section{Security for girls in camps}

Respondents mentioned that girls are much more secure in Bangladesh compared to Myanmar. Fear of abduction and sexual harassment by military is reported to be nonexistent in the camps. They feel more secure after coming to Bangladesh.

"Here (camp) we do not have any security threat. We can sleep peacefully at night. Unlike in Myanmar, girls are much safer here (camp)." (IDI 5, Married woman, age 19)

"Girls are in more secure in camp. We were in a disastrous situation there (Myanmar), but here (camp) we are living in peace. There, our girls had to wear four layers of clothes so that the army cannot undress them easily. If one layer of clothing is torn down, there are other layers of protection. Parents used to teach their daughters to wear dresses in such a way that it would be difficult to undress them." (Majhee, FGD Participant)

Although incidences of sexual harassment inside the camp was not been reported by any respondent, feeling of insecurity has been shared by some respondents.

"My husband is not present here. There is no male in our house. I'm living here with my two children and my younger sister. So l've always a fear of getting molested or being robbed by an intruder, or if someone steals my children." (IDI 6, Married girl, age 20)

“Couple of months ago a girl from my neighbor's house (inside camp) was abducted by some bad people but they got caught by the army and couldn't take the girl away. But there is fear of such incidence." (IDI 30, Married boy, age 23)

\section{Risk of trafficking, forced or transactional sex}

Respondents were asked if they are facing any security threats or risks such as trafficking, drug abuse, involvement in illegal activities, forced sex, transactional sex and/or any other issue in camps. In general, none of the study respondents reported to be victim of any such event.

A majority of the respondents mentioned that trafficking, forced/transactional sex are not prevalent in camps. Only a few mentioned that they had heard of such incidents happening in a different camp at the time of their arrival. However, the situation has since improved, and currently no such security threat in camp. Respondents, however, mentioned some exceptional cases when asked about trafficking and transactional or forced sex.

"At the beginning when we came here, I heard that young boys, girls, and women were being sold for money... Nothing like this is occurring at present. One day, a man came to my mother, and said, 'Give me your daughter. I will arrange a job for her in a garment factory in Dhaka. I will marry her.' My mother understood that the man was a human trafficker. She told him that she had left her country for the sake of her daughters. So, she wouldn't send them to work outside the camp. She would rather feed them from what the government is giving. Nowadays, girls have become smart. So they don't fall prey to such offers." (IDI 15, Unmarried girl, age 17)

\section{Risk of illegal activities by young people}

To better understand life in the camps, we wanted to understand youth and adolescent perceptions of illicit or illegal activities. Almost all of the respondents stated that in the camps, there are restrictions on 
individual mobility and people are under surveillance by authorities within the camps. Therefore, most Rohingya, including young people, have limited opportunities to get involved in illegal activities. Religious leaders also play a role in maintaining law and order and motivate people against any wrongdoing.

“How can people get involved in any illegal activities here (inside camp)? People can't move from one block to another freely. People can't go out of their homes after Isha (evening prayer). We have volunteers here who keep watch on the neighborhood day and night. Each Majhee has 5 volunteers under him who are actively vigilant. How can anyone get involved in illegal activities under such surveillance? Young boys can't roam around at night, and girls are not allowed to talk to strangers. No one can move around within the camps." (IDI 13, Unmarried woman, age 20)

\section{Risk of substance abuse}

A majority of the respondents denied the use of drugs among the Rohingya. Some respondents mentioned that the use of drugs has decreased in camp compared to in Myanmar due to changed living conditions.

Here, the supply of drugs is less, but prices are much higher. Also, in the camps, there is no private place where people can take drugs in secrecy; because of the cramped housing pattern, they are unable to use drugs." (IDI 40, Unmarried boy, age 14)

\section{Environmental hazard}

Respondents mentioned they are concerned about environmental hazards in the camps. Living in makeshift houses on top of hills is risky, especially during the monsoon season. The risk of heavy rain resulting in floods and landslides threaten their lives in camp.

"When we first came here during the dry season, everything was accessible and convenient, but since the 'barish' (rain) started everything has become very difficult, our houses have been flooded. We have been living in great difficulty and pain since then. We are living in hardship as our houses are damaged during the storms, and the ground is flooded. Since this room has a cement floor it's a bit dry, but we cannot sit in the other room at all. Even after all these troubles, the government or anyone else don't understand our pain." (IDI 29, Married man, age 21)

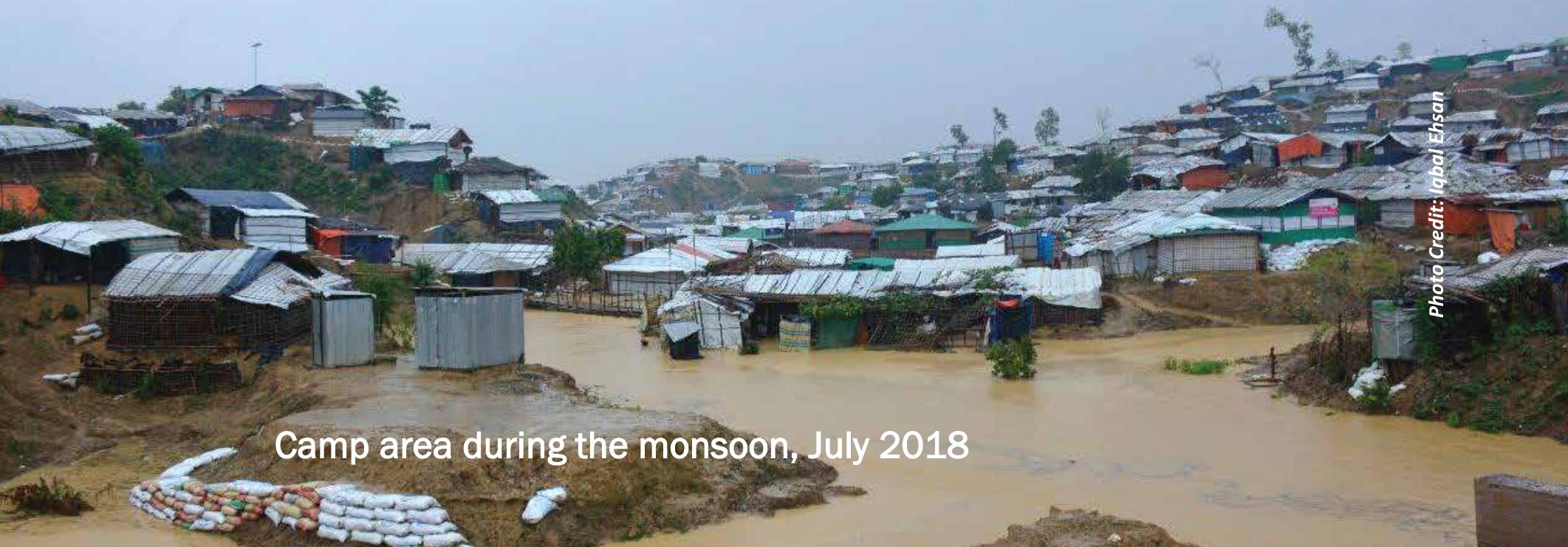




\section{Conflict and dispute resolution}

There were a few reports of conflict over money and power within the camps. Respondents reported that disputes are resolved first by the Majhee(s) with the help of the volunteers. Administrative units, each comprising of a Majhee and volunteers, have been set up for overall governance of the camps. In certain cases when disputes cannot be resolved by volunteers or Majhee(s), the Bangladesh authority is called in to help minimize disputes and tackle any potential conflict.

“Here (camp) if any conflict arises, then the police will come and resolve it. Here, the number of people from Buthidung is much higher than the number of people from Maungdaw. Buthidung people sometimes create chaos in camp. They try to dominate us." (IDI 30, Married man, age 22)

"When any dispute breaks out in camp, the elders of the community and the Majhee(s) resolve those ... We have 5 administrative committees. These committees resolve all type of problems in camp. When the committee fails to resolve a dispute, the case is transferred to the CiC office for further action and solution." (Majhee, FGD Participant)

\section{FUTURE ASPIRATIONS}

Of particular interest is how respondents, particularly young people, view their future opportunities and whether they aspire to return to Myanmar or remain in Bangladesh. Adolescent and young Rohingya respondents were asked about their thoughts on repatriation, educational aspirations, and desire for involvement in income generating activities.

\section{Repatriation}

Almost all respondents expressed a desire to go back to Myanmar. However, their return was contingent upon a number of preconditions including acquiring citizenship status and having their rights restored.

"That (Myanmar) is our country. Do you think we are happy to live like this here (camp)? We are not at peace here. There (Myanmar) we had our ancestral lands, assets, money; we could earn our livelihood. But here we sit idle without any work. We couldn't bring any money with us, so how are we going to survive?" (IDI 39, Unmarried boy, age 17)

"Why would I return there (Myanmar)! If we have to return to that country, we have to be given freedom to move anywhere, which we didn't have before. All of our wealth and properties have been lost. So, if we have to stay there, our homeland has to be returned to us in our own way. Otherwise we won't return there. What's the benefit of migrating from one country to another if we have no place to live! Not only do we want our homeland, but the wealth and property that we've lost also has to be returned to us." (IDI 10, Married woman, age 20)

\section{Educational aspirations}

Both married and unmarried respondents were asked if they wanted to pursue education in the camp if the opportunity existed. In response, a majority of respondents, both boys and girls, expressed a desire to obtain education. Respondents said they would study if they were given the opportunity in camp. Respondents who were studying in Myanmar wanted to resume their studies in camp. FGDs with Rohingya adult women, Majhees and Imams indicate they aspire to provide the young Rohingya generation with education for the betterment of their future.

"I want to study further. I want to study up to class ten. I would look for jobs that are eligible for a class 10 graduate" (IDI 37, Unmarried boy, age 17)

"The schools in the camps are not focusing much on studies; rather, students are playing in these schools. If there are high schools ... it would have been better." (Majhee, FGD participant) 


\section{Involvement in income generating activities}

Almost all the respondents said that they wanted to get involved in income generating activities. They want to earn money to survive and to live a better life. They want skill-building and livelihood training to generate earnings. Almost all adolescents and women respondents, both married and unmarried, want to earn their living through tailoring and sewing. Some mentioned that they want to earn through small handicrafts business-weaving carpets, making caps, etc.

"I can make handicraft items with bamboo. But I'm not very skilled at it. I want to learn to make utensils with bamboo or to sew clothes." (IDI 6, Married woman, age 20)

"I know sewing. I can do embroidery, I can weave carpets, I know almost all handicrafts. I took a loan to buy a sewing machine after coming here. I earn some money from sewing, which covers my daily expenses." (IDI 9, Married woman, age 22)

Findings indicates that male respondents have limited opportunities to work and earn inside the camps by engaging in site development activities such as, day laborer, mason, and carpentry. Male respondents also expressed their interest in receiving training for income generation.

"I want to have training to become a sales man at a pharmacy. I used to work in a pharmacy in Cox's Bazar. But after a month I had to go back to Myanmar because of my mother's illness. If I had been working in that pharmacy, I would have learnt many things by now. I have two desires, one is to work with an NGO and another is to learn pharmacyrelated work." (IDI 27, Married man, age 21)

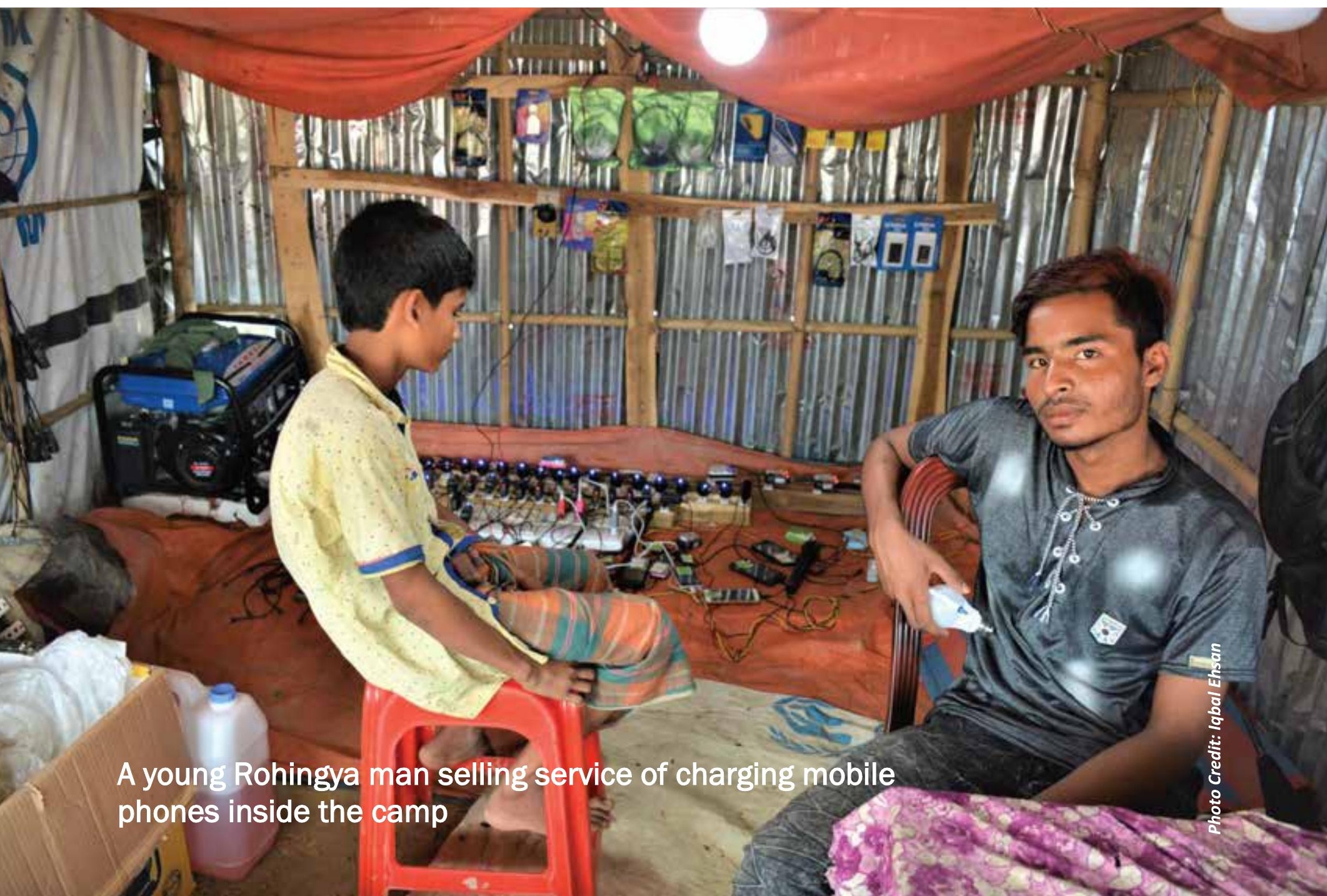




\section{CHAPTER 7: EXPERIENCE OF THE HOST COMMUNITY}

Large scale refugee movements have economic and social impacts on their host community. The problem is exacerbated when the receiving community itself has limited resources. This study explored the experience of the host community in Bangladesh, specifically their perceptions, concerns, and their view of the changing dynamics of their lives after the latest mass arrival of the Rohingya since August 2017. From FGDs held in the host community, we found that in the early stages, the host community was welcoming and sympathetic towards the Rohingya's plight and extended their support by giving shelter, sharing food, and providing clothes to the Rohingya people who had just escaped deadly ethnic violence in their home country. However, respondents report that gradually, their perception and attitude towards the Rohingya are changing to be negative.

"We will be in much better living conditions if the Rohingya no longer stay in our area. Our youth can be saved from drug, theft, and robbery which increased after their arrival. Agricultural productivity of the area will increase. Amount of fish will increase in the river in this region." (Married man, FGD participant, host community)

"They should be sent back to Myanmar. But their repatriation should be done in such a manner where they will no longer face any oppression. Every country should come forward to resolve this crisis and repatriate them. It is an emergency for us and also for them (Rohingya)." (Unmarried girl, FGD participant, host community)

\section{REDUCED LIVELIHOOD OPPORTUNITIES}

The regular life and livelihood of the host community have been reported to be adversely impacted with the presence of the Rohingya in the region. Many people living in the area have historically depended on forestbased livelihood options such as wood cutting and selling. Respondents reported that forest area in their locality has been cut down to make space for Rohingya settlements which has depleted natural resources and limited the livelihood of the locals.

"Before their (the Rohingya) arrival, poor people of the area used to earn their livelihood through agriculture, cultivating land, fishing in the river, and cutting wood from the forest. But after their arrival we don't have these options anymore." (Married man, FGD participant, host community)

"Earlier people could earn their living from the forest, but now they can't go there because camps have been established on top of the hills. People who used to work as day laborers are not getting work now because the Rohingya are taking it away by working at a much lower rate. Bengalis used to work at 500 taka per day. But the Rohingya are working for 200-300 taka a day. So they are getting all the work. Now Bengalis are dwelling in poverty." (Unmarried girl, FGD participant, host community)

The host community mentioned that overpopulation in the area has led to shortages in the supply of food and other resources. At the same time, the higher demand for food and services is increasing the price of all items.

"Earlier we used to buy $1 \mathrm{~kg}$ fish for 50 taka. Now we have to pay 200 taka for the same amount of fish. Vegetables that we bought for 25 taka per kg, we now buy for 50 taka per kg." (Unmarried girl, FGD participant, host community) 


\section{EDUCATION AT RISK}

Respondents feel that the arrival of the Rohingyas has disrupted the education of their children. There is a shortage of school teachers who are taking jobs in NGOs working in the camps. Organizations working with the Rohingya need educated people for their program implementation and other activities. Their compensation package is higher than a teacher's salary. Hence, teachers are leaving the schools to join NGOs.

"There is teacher shortage in the schools. Teachers are joining NGOs with higher salary and leaving behind the teaching profession." (Unmarried girl, FGD participant, host community)

Secondary-level students can also get employment with a good salary in camp-based NGOs. As a result, students prefer getting jobs in camps and are discontinuing their studies.

"Our children are getting derailed for money. Boys and girls who were attentive at studies are now more interested to earn money by working in the camp (camp-based NGOs). Students who couldn't earn even 100 taka a day are now earning 500 taka by working in camp. As a result many of the students have left school and are taking jobs in camp." (Unmarried boy, FGD participant, host community)

\section{DISEASE PATTERN AND HEALTHCARE SERVICES}

Inadequate water sanitation system, congested living conditions, and lack of cleanliness have increased adverse health effects as mentioned by host community respondents. Outbreaks of diseases such as skin disease, diphtheria, diarrhea, and malaria are growing in the area after the arrival of the Rohingya.

"Now we observe different diseases around. Diarrhea is now common. And the outbreak of another disease called Diphtheria is prevailing at present. We never heard of this disease earlier, but now we are taking vaccine for it. We are also taking medicines for cholera after the arrival of the Rohingya." (Married man, FGD participant, host community)

Respondents mentioned that after the arrival of the Rohingya, the number of health facilities and activities of health workers have increased in the area. In the past, these were very limited. After the latest arrival, availability of health services increased due to activities by different agencies. Respondents mentioned that maternal health service has increased, which is a positive development. But the respondents also reported that in hospitals and other health facilities, the Rohingya are given priority over Bangladeshis in service delivery. Bangladeshis have to wait for longer hours for any healthcare service.

"There was favorable situation to get medical checkups before the arrival of the Rohingya, it has decreased now. Transportation was easier before, but now because of the Rohingya, we are facing problems in arranging transportation. Moreover, fare has increased a lot. The security checking at each check post also takes more time than before. We cannot even go to hospital in an ambulance in case of emergency, as we have to go through security checking at each check post. This is highly risky for a pregnant woman as often, it delays reaching the hospital. There are many healthcare centers here where the Rohingya are given priority. We need separate healthcare centers for the local people. We don't want to get service at the same places as the Rohingya." (Unmarried girl, FGD participant, host community)

\section{CHANGES IN MARRIAGE PRACTICE}

A majority of the Rohingya respondents mentioned that the Rohingya don't marry outside their community and denied the incidence of marriages between the Rohingya and the Bangladeshis. However, FGDs with the host community suggest that Bangladeshi men are marrying Rohingya girls living in the camps. A majority of host-community respondents reported that after the arrival of the Rohingya, the practice of polygamy has increased in the area. Both married and unmarried men, but mostly married men, are marrying Rohingya girls as marriages can be performed upon verbal consent only without any formal registration inside the camps. Respondents mentioned displacement and crisis has left Rohingya girls in a vulnerable state. Hence Rohingya families are arranging marriages with Bangladeshi men irrespective of their marital status. Host community respondents said that Bangladeshi men are marrying Rohingya girls because they are beautiful and also because marrying a Rohingya girl doesn't incur any expense. Rohingya 
families are entitled to food assistance so the men don't bear additional expenses; rather, Bangladeshi men can avail themselves of a share of relief/ration of the Rohingya girl's family.

"Bangladeshi men are marrying Rohingya girls even when they have wives and children.. Marriage registration is not required in camp. Marriages are taking place on verbal consent. Multiple marriages have increased in our area as Bangladeshi men are taking multiple wives from different camps. They don't have to bear their expenses as Rohingya girls are entitled to ration." (Unmarried girl, FGD participant, host community)

"My husband has affairs with two different Rohingya women living close to our home. When I discovered my husband's affairs, I told them to keep away from my husband. I have two children, I don't know what will happen in future. Nowadays, my husband often behaves badly towards me. I don't want to see Rohingya in our country anymore." (Married women, FGD participant, host community)

\section{SOCIAL INSECURITY}

Some host community respondents allege that Rohingya girls are getting involved in transactional sex with Bangladeshi men in and around the camps. Respondents also perceive that the risk of STIs, including HIV, has increased among local boys.

"There are many girls in the locality who are engaged in sex in exchange of money/transactional sex. Many married and unmarried men of our area are engaging in physical relationships with them. These girls belong to both the recent and earlier Rohingya population living in the area." (Unmarried boy, FGD participant, host community)

The host community feels that living next to a large number of Rohingya is a security concern for their unmarried daughters. Respondents report that there is a strong possibility of sexual harassment of local girls by Rohingya boys and young men on the street. Respondents also said they are worried about local girls working in the camps. They fear that the Rohingya might perpetrate violence against their daughters.

Host community respondents said that the risk of illegal activities has increased in the area with young boys being offered money by the Rohingya for smuggling and drug peddling. Substance abuse was reported to have also increased.

"The risk for young people in getting involved in illegal activities in exchange for money has increased after the recent arrival. Local boys are engaging in drug peddling in exchange of money." (Unmarried girl, FGD participant, host community)

Respondents said that the Rohingya are involved in drug smuggling. They are carrying drugs from Myanmar to Bangladesh and selling them to the young people of the area.

"Rohingya people carried Yaba tablets from Myanmar during their latest arrival here. They sell tablets (Yaba) in camps. Young boys of our area are buying drugs from camp and are also using drugs inside the camps. When they don't have cash, they sell cattle to arrange for money to buy Yaba from the Rohingya." (Unmarried boy, FGD participant, host community)

\section{RISK OF COMMUNITY CONFLICT}

Findings suggest that in general, the host community perceives the Rohingya to be of an aggressive nature. In FGDs with the host community, we repeatedly encountered descriptions of conflicts with the Rohingya and the local people's fear of being threatened and beaten by the Rohingya. The host community also fears getting outnumbered by the Rohingya.

"The problems that arose after their arrival are uncountable. We are suffering from 1991, but this pressure had increased in 2017-2018. If any conflict takes place between Rohingya and Bengalis, then only we will suffer, we won't be able to retaliate. This because we are two and they are two hundred." (Married man, FGD participant, host community) 
"If an argument arises with the Rohingya, their relatives come up with weapons to beat us. They don't acknowledge that Bangladesh has given them shelter. Rohingya say foreigners are providing us food. This is now our country. We have bought this land. They say we have come here and that is why you are getting food and all these facilities." (Unmarried girl, FGD participant, host community)

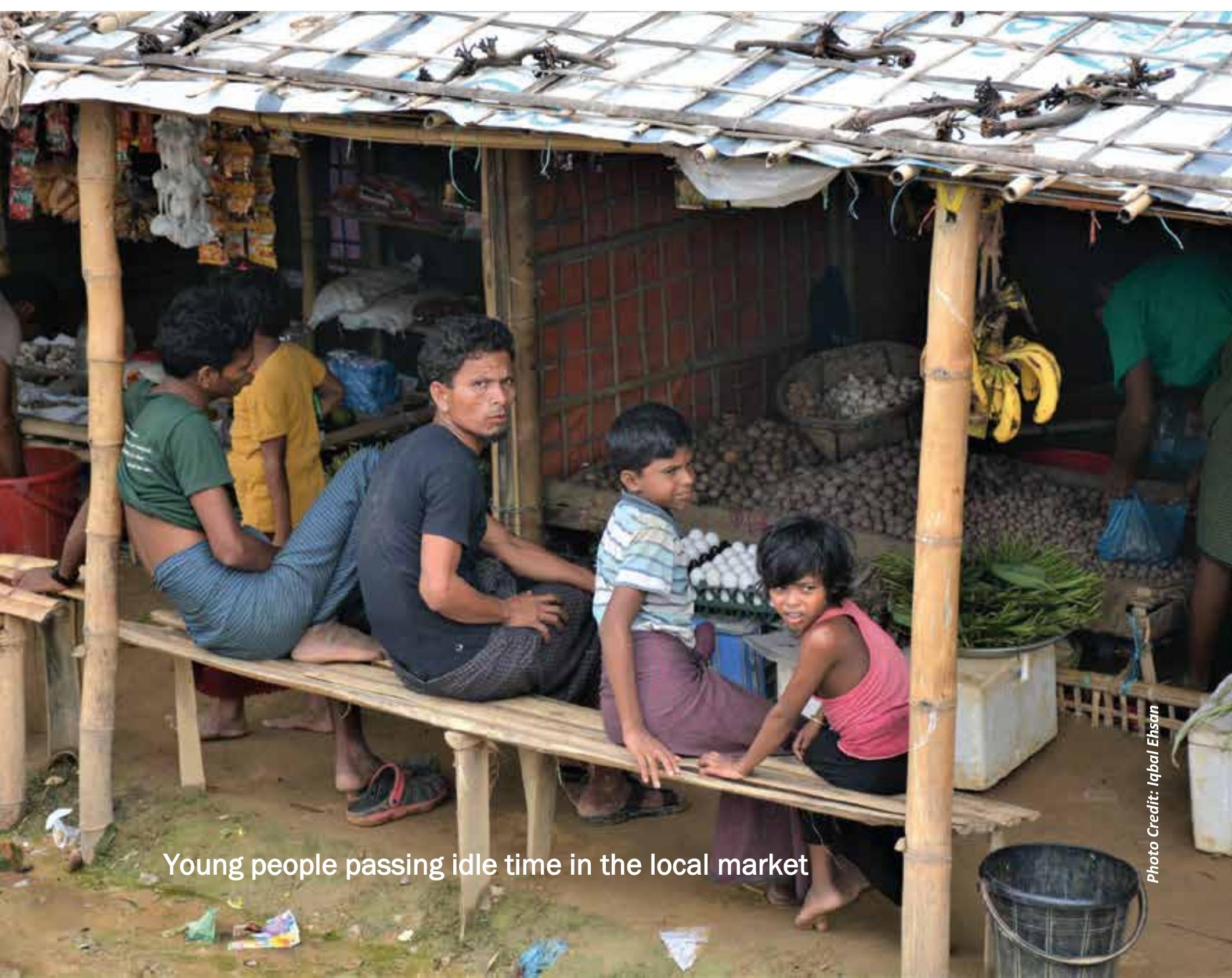




\section{CHAPTER 8: DISCUSSION AND RECOMMENDED ACTIONS}

The findings from this study provide essential insights into the lives of the Rohingya living in the camps in Bangladesh. This chapter summarizes the findings from IDIs with Rohingya adolescents and youth, service providers and program personnel from the public sector, and national and international NGO staff working with the Rohingya population, as well as FGDs with Rohingya community and religious leaders and members of the surrounding host community.

We expect that findings from this formative research and recommended actions will help the Government of Bangladesh, donors and other relevant stakeholders to mobilize resources and deliver SRH services in a more organized and efficient way and thus help to ensure the well-being of the Rohingya adolescents and youth.

\section{FINDINGS}

\section{Child marriage is on the rise in the camps}

Marriage registration is lax inside the camps; in the absence of any legal procedures and restrictions on the minimum marriageable age, child marriages will likely rise among the Rohingya. It is found that the Rohingya traditionally prefer early marriages. Conditions within the camps also inadvertently reinforce early marriages; for example; marriage leads to household separation and additional ration cards. Traditional Islamic beliefs, as well as a desire to preserve girls' sexual purity, are factors that motivate parents to arrange marriages for girls. We found that the exchange of dowry was practiced among the Rohingya in Myanmar and is still prevalent despite the constrained economic conditions in the camps.

\section{Marriage dynamics are changing in the surrounding host community}

In interviews with the Rohingya in the camps, we did not hear much about marriages between the Rohingya and the Bangladeshis. However, in focus group discussions, host community members reported marriages between Rohingya girls and Bangladeshi men. This contradictory finding may be explained by the fact that the Rohingya are unlikely to report such marriages because they are against the law. ${ }^{8}$ Also, if the Rohingya leave the camp after marriage to live among the host community, these marriages become more visible to the host community, Further, host community members allege that Rohingya girls are involved in transactional sex in and around the camp, and they also attribute an increase in the incidence of polygamy to the presence of Rohingya.

\section{Large families are preferred by the Rohingya}

Findings indicate that there is high demand for children and large families are preferred by the Rohingya. Religion plays a major role in the preference for large family size. The most dominant and common response regarding desired family size was "Allah's will" and /or "as many as Allah gives." Findings suggest that economic interest also plays a role in preference for large family size-it is believed that more children will bring prosperity.

\section{Use of contraception is limited among the Rohingya}

Knowledge and use of contraception are limited among the Rohingya. Injection (Depo-Provera) and oral pill ("card dabai" in Rohingya dialect) are the two most popular contraceptive methods among them. Respondents reported that husbands and mothers-in-law are the primary gatekeepers and decision makers for contraception use among women and girls. Religious barriers are reportedly the main reason for non-use of contraception which is viewed as 'haram' and 'sin' and considered prohibited by Islam. We also found that the association of contraceptives with immorality is common among the Rohingya. Respondents noted that the use of contraceptives for health reasons is acceptable.

\footnotetext{
8 Since 2014, the Bangladesh government banned marriages between Rohingyas and Bangladeshi nationals and issued a gazette notifying marriage registrars not to register any marriage without ensuring that both the bride and groom are Bangladeshi nationals (Prodip, 2017).
} 


\section{Misinformation about sexual and reproductive health issues is widespread}

Misconceptions and rumors hinder the Rohingya women's access to and use of family planning products and services. In particular, fear of contraceptive side effects including infertility and even death keep many women from choosing effective methods. Misinformation is propagated by word-of-mouth from neighbors, friends and family, and elderly women in the community. Fear and misconception regarding institutional delivery are also prevalent among the Rohingya.

\section{Reproductive health services are often available but inaccessible to Rohingya adolescents and youth}

The deprivation of services that the Rohingya experienced in Myanmar shapes their service-seeking behavior in Bangladesh. During interviews and FGDs, most of the respondents stated that they were not allowed to go to hospitals in town due to their restricted mobility in Myanmar. They reported being largely dependent on local pharmacists and traditional healers to meet their health needs. In the camp, despite the availability of health services, there is a limited utilization of services by Rohingya adolescents and youth. Traditionally, girls and women are less mobile in the Rohingya community, and they are not encouraged to leave home and travel to the health facility. This reduced mobility coupled with transportation issues, including poor road conditions in the camps, were identified by service providers as the reasons for limited uptake in services by Rohingya adolescents. Program personnel and service providers also highlighted that adolescent boys hardly visit health facilities for SRH in the camps.

\section{Host community members perceive the Rohingya both as social and economic threats}

FGDs with host community members reveal tension between the host community and the Rohingya living in camps. Host community members allege that Rohingya men and boys are involved in illegal activities including violence, smuggling, and drug peddling. Host community members also expressed fear of harassment of local girls by Rohingya men. In addition to their concern about deforestation, the host community also emphasized increased food prices and reduced livelihood options as concerns. We heard from host community members that they feel ignored by the NGOs and service providers, and that the Rohingya are prioritized in health facilities.

\section{RECOMMENDED ACTIONS}

\section{Engage community members and religious leaders in designing programs}

Traditional Islamic beliefs, specifically the high demand for children and preference for large families may be the primary hurdles to the promotion of family planning. A limited number of the respondents (including a religious leader) approved of the use of contraception on health grounds. The identification of avenues such as contraception for the health benefit of mothers and children is important for making inroads against the resistance to contraceptives. Engagement of Imams and religious leaders is vital as they have significant influence in shaping the community's belief systems and corresponding behaviors. Given that traditional cultural beliefs and practices impede access to healthcare, community health education need to be prioritized and different set of actors from Rohingya community need to be engaged to reach out to different groups of population.

\section{Raise awareness among elder Rohingya women about sexual and reproductive health issues and engage them as agents for positive change}

Decisions about reproductive health for adolescent girls and women are often guided by the experiences and advice of female elders in their community. Information shared by female elders may be valued more than that of a medical professional. It is harmful if older women are promoting ineffective, misleading, or inaccurate information and rumors. Respected elders should be engaged and empowered to provide accurate information about SRH issues through targeted training and resources. Engaging this population may also open an avenue to work with mothers-in-law who play a crucial role in girls and women's decisions regarding contraceptive use.

\section{Working with husbands is crucial in promoting contraceptive use}

Culturally, the Rohingya is a male dominant community and women are expected to obey their husbands when it comes to childbearing and fertility decisions. Effective ways to work with husbands to promote contraceptive use must be explored. Additionally, another influential male group, community leaders such as Majhees, should be mobilized to work with husbands to provide accurate information and encourage them that the use of contraceptives may be beneficial for both their wives and their offspring. 


\section{Expand efforts to improve adolescent sexual and reproductive health}

The global community increasingly realizes that working with and prioritizing adolescents is an effective strategy for improving overall population health currently and for future generations. In this study, our findings indicate the Rohingya adolescents and youth have changed some perceptions of contraceptive use after migration to Bangladesh due in part to increased contact with service providers. Steps should be taken to better inform Rohingya adolescents and youth about the availability of SRH services in their localities. Health education tools should be responsive to low literacy levels among the Rohingya and use strategies to provide information about STIs, HIV, and alternative methods of contraception.

\section{Reach out to adolescents rather than waiting for adolescents to reach out}

Our findings suggest that mobility of Rohingya adolescent girls is restricted and traditional gender norms, shyness, and transportation issues make it difficult for adolescents to access facilities. Findings also indicate that despite the availability of the SRH service points, male adolescents, especially unmarried boys, hardly visit these facilities. Mobile outreach may be a viable and effective option for reaching out to adolescents in the camps. Engaging Rohingya volunteers along with Bangladeshi service providers can be a promising combination in these efforts.

Invest in safe spaces to increase girls' social interaction and sense of safety in camps

Restricted mobility and concerns about sexual safety can affect behaviors with respect to access to services, ability to earn livelihoods, and motivate child marriages. Safe space model can be used to increase social interaction and networking and mobility among adolescent and young Rohingya girls within the camps. If these spaces offer opportunities to access productive assets and work as network nodes for outreach activities, the welfare of girls and their families would also potentially improve.

\section{Address the needs and insecurities of the host community}

Findings suggest that host community members feel marginalized, and they state that their needs have been compromised after the arrival of the Rohingya. The changing social and economic dynamics of the host community after the arrival of the Rohingya and its impact on the host community need to be assessed and incorporated while designing interventions. 


\section{REFERENCES}

ACAPS.2017. Rohingya Crisis Situation Analysis November 2017. Available at: https://reliefweb.int/report/bangladesh/rohingya-crisis-situation-analysis-november-2017

Ainul, Sigma and Sajeda Amin. 2015. Early Marriage as a Risk Factor for Mistimed Pregnancy among Married Adolescents in Bangladesh, Asia-Population Journal, 30 (1) pp 7-34.

Ainul, Sigma, Ashish Bajracharya, Laura Reichenbach, and Kate Gilles. 2017. "Adolescents in Bangladesh: A Situation Analysis of Programmatic Approaches to Sexual and Reproductive Health Education and Services," Situation Analysis Report. Washington, DC \& Dhaka, Bangladesh: Population Council, The Evidence Project.

Amnesty. 2017. "Caged without a Roof" Apartheid in Myanmar's Rakhine State

Bangladesh Bureau of Statistics (BBS) and Statistics and Informatics Division (SID) 2012. Bangladesh Population and Housing Census 2011: Socio-Economic and Demographic Report.

Bruce, Judith and Shelley Clark. 2004. "The implications of early marriage for HIV/AIDS policy," brief based on background paper prepared for the WHO/UNFPA/Population Council Technical Consultation on Married Adolescents. New York: Population Council.

CARE Bangladesh. 2017. Myanmar Refugee Influx Crisis from August 2017. Rapid Gender Analysis Report.

Chowdhury, M.A.K., S.M. Billah, F. Karim, A.N.S. Khan, S. Islam, and S.E. Arifeen. 2018. Demographic Profiling and Need Assessment of Maternal and Child Health (MCH) Care for the Rohingya Refugee Population in Cox's Bazar, Bangladesh. Dhaka, Bangladesh: International Centre for Diarrheal Disease Research, Bangladesh (icddr,b).

Directorate General of Health Services (DGHS), 2018. Ministry of Health and Family Welfare, Government of Bangladesh. Data. Rohingya Undocumented Myanmar Nationals (UMN) program. Available at: http://103.247.238.81/webportal/pages/controlroom_rohingya.php

Girls Not Brides. 2016. Child Marriage in Humanitarian Crises. https://www.girlsnotbrides.org/wpcontent/uploads/2016/05/Child-marriage-and-humanitarian-crises-June-2016.pdf

Hickox. K. 2009. Myanmar: Delivering Care to Isolated Rohingya. July 23, 2009, Amsterdam: Medecins Sans Frontieres.

Huemer, Julia, Niranjan Karnik, Sabine Voelkl, Elisabeth Granditsch, Kanita Dervic, Max Friedrich, and Hans Steiner. 2009. Mental Health Issues in Unaccompanied Refugee Minors, Child and Adolescent Psychiatry and Mental Health, 2009, 3:13

Inter Sector Coordination Group. 2018. Situation Report: Rohingya Crisis.

Inter Sector Coordination Group. 2017. Situation Report: Rohingya Refugee Crisis.

IOM Bangladesh (International Organization for Migration). 2018a. Rohingya Refugee Crisis Response; External Update 19-25 January 2018. https://reliefweb.int/report/bangladesh/iom-bangladeshrohingya-refugee-crisis-response-external-update-19-25-january-2018

IOM Bangladesh 2018b. Needs and Population Monitoring (NPM) Site Assessment: Round 11. Download link: https://www.humanitarianresponse.info/en/operations/bangladesh/assessment/needs-andpopulation-monitoring-npm-bangladesh-round-11-site

Mahmood, Syed et al. 2017. The Rohingya People of Myanmar: Health, Human Rights, and Identity. Lancet, Volume 389, Issue 10081, P1841-1850, May 6, 2017.

Medecins Sans Frontieres. 2013. Myanmar: Violence and Intimidation Leave Tens of Thousands without Medical Care. Amsterdam Medecins Sans Frontieres. 
Mourtada, Rima, Jennifer Schlecht, and Jocelyn Dejong, 2017. A Qualitative Study Exploring Child Marriage Practices among Syrian Conflict-Affected Populations in Lebanon. Conflict and Health, 11.

Myo, Myint. 2013. Final Report of Inquiry Commission on Secretarian Violence in Rakhine State. Myanmar. Republic of the Union of Myanmar. http://www.burmalibrary.org/docs15/Rakhine Commission Report-en-red.pdf

NPM-ACAPS analysis hub. 2018. Rohingya Crisis: Host Community Review. January 2018.

https://www.humanitarianresponse.info/sites/www.humanitarianresponse.info/files/assessments/18013 1 host communities.pdf

OHCHR (Office of the United Nations High Commissioner for Human Rights). 2018. Report of the Independent International Fact-Finding Mission on Myanmar.

Oxfam. 2018. One Year On: Time to Put Women and Girls at the Heart of the Rohingya Response. Oxfam Briefing Paper. September 2018.

Population and Housing Census of Myanmar 2014, Provisional Results, Department of Population, Ministry of Immigration and Population, August 2014.

Prodip, MA. 2017. Health and Educational Status of Rohingya Refugee Children in Bangladesh. Journal of Population and Social Studies, 25 (2), pp 135-146.

RRRC and ISCG, 2018, One year joint press release. https://www.humanitarianresponse.info/en/node/169321

Save the Children. 2018. "Horrors I Will Never Forget” The Stories of Rohingya Children. https://resourcecentre.savethechildren.net/node/12501/pdf/horrors-report.pdf

Save the Children. 2013. Unspeakable Crimes against Children: Sexual Violence in Conflict. https://resourcecentre.savethechildren.net/node/7252/pdf/7252.pdf

Santhya, K.G. 2011. "Early Marriage and Sexual and Rreproductive Health Vulnerabilities of Young Women: A Synthesis of Recent Evidence from Developing Countries." Current Opinion in Obstetrics and Gynecology, (23), pp 334-339.

Santhya, K.G. and S.J. Jejeebhoy. 2007. "Early marriage and HIV/AIDS: Risk Factors among Young Women.” Economic and Political Weekly, 42(14):1291-1297.

Sen, Amartya. 2008. "Violence, Identity and Poverty.” Journal of Peace Research, 45(1), 5-15.

Sheehy, G, Y. Aung, C. Siestra, A. Foster. 2015. Near the City but Hard to Reach: A Reproductive Health Needs Assessment in Peri-Urban Yangon. Cambridge, MA: CRHC.

Translators Without Border (TWB). 2017. Rohingya Zuban. Available at: https://translatorswithoutborders.org/rohingya-zuban/ Accessed September 19, 2018.

UN Women (United Nations Entity for Gender Equality and the Empowerment of Women). 2017. Gender Advocacy Paper for Rohingya Refugee Crisis Response in Bangladesh.

UNHCR (United Nations High Commissioner for Refugees). 2007. Analysis of Gaps in the Protection of Rohingya Refugees.

UNICEF (United Nations International Children's Fund). 2018. Lives in Limbo: No End in Sight to the Threats Facing Rohingya Children.

UNICEF (United Nations International Children's Fund). 2017. Humanitarian Situation Report No.14 (Rohingya influx).

UNFPA (United Nation Population Fund). 2018. UNFPA Rohingya Humanitarian Response. Monthly Situation Report. Available online: http://bangladesh.unfpa.org/sites/default/files/pubpdf/UNFPA_SitRep_External_16-March percent202018 percent20Final.pdf 
UNFPA (United Nation Population Fund). 2016. Adolescent Girls in Disaster \& Conflict: Interventions for Improving Access to Sexual and Reproductive Health Services. https://www.unfpa.org/sites/default/files/pub-pdf/UNFPA-Adolescent_Girls_in_Disaster_ConflictWeb.pdf

UNFPA (United Nation Population Fund) and Save the Children. 2009. Adolescent Sexual and Reproductive Health Toolkit for Humanitarian Settings. https://unfpa.org/sites/default/files/pubpdf/UNFPA ASRHtoolkit english.pdf

World Health Organization. 2018. News Release. http://www.searo.who.int/mediacentre/releases/2017/1666/en/ Accessed April 2, 2018.

World Health Organization. 2018. Rohingya Refugee Crisis in Cox's Bazar, Bangladesh Health sector bulletin 3. http://www.searo.who.int/bangladesh/healthsectorcxbbanbulletinno3.pdf Accessed April 02, 2018.

World Health Organization. 2016. Global Health Estimates 2015: Deaths by Cause, Age, Sex, by Country and by Region, 2000-2015. Geneva: WHO.

Women's Refugee Commission. 2016. A Girl No More: The Changing Norms of Child Marriage in Conflict, https://www.womensrefugeecommission.org/refugees/adolescent-girls/research-and-

resources/1311-girl-no-more 


\section{ANNEX 1: GLOSSARY OF CHITTAGONIAN/ROHINGYA DIALECTS}

\begin{tabular}{|c|c|c|c|c|c|}
\hline English & Bengali & $\begin{array}{l}\text { Chittagonian/ } \\
\text { Rohingya dialects }\end{array}$ & English & Bengali & $\begin{array}{l}\text { Chittagonian/ } \\
\text { Rohingya dialects }\end{array}$ \\
\hline Child & শিশ & গুরা ফোয়া & Medicine & ওযুধ/চিকিৎসা & দারু \\
\hline $\begin{array}{l}\text { Mental } \\
\text { Exploitation }\end{array}$ & মানসিক নির্যাতন & মনত হষ্ট, জালায়ে & Rape & ধর্ষণ & জুলুম, রেপ \\
\hline Victim & শিকার হয়েছেন & মুখোত পজ্জে & Good, Well & ভালো & গম \\
\hline Safe & নিরাপদ & হেফাজত & Safely & নিরাপদে আছে & হেফাজতে আছে \\
\hline Bad People & খারাপ মানুষ & আলেকিন & Traffickers & পাচারকারী & ফোয়া ধরনি \\
\hline Addiction & মাদক গ্রহন করে & নেশা হায়, নেশা গরে & For Money & টাকার জন্য & টেয়ার লায় \\
\hline Future Plan & ভবিষ্যৎ পরিকল্পনা & পরে কি গরিবা & $\begin{array}{l}\text { Previous } \\
\text { occupation }\end{array}$ & আগে কি করতেন & আগে কি গত্ত্যেন্ \\
\hline Handicrafts & হস্ত শিল্প & আঁতর হাম & Chance & সুযোগ & মওকা \\
\hline Query & জিজ্ঞেস & উুসার & Rules/Rituals & নিয়ম রীতিনীতি & রসম/রসন \\
\hline Quick & তাড়াতাড়ি & স-রে স-রে & Friend & বন্ধু & ওঁয়াজ্জা \\
\hline Pregnant & গর্ভবতী & হামিল, পোয়াতী & Fear & ভয় & ডর \\
\hline Harm & ক্ষতি & খতারা & Spread, Infect & কেমন করে ছড়ায় & ক্যান গরি অয় \\
\hline $\begin{array}{l}\text { Avoid to } \\
\text { addiction }\end{array}$ & $\begin{array}{l}\text { নেশা থেকে বিরত } \\
\text { থাকার জন্য }\end{array}$ & নেশাত্তুন বাঁচিবাল্যায় & Tensed & চিন্তিত & ট্যানশনত \\
\hline Family & ঈরিবার & ঘর, ঘরত্ & Where & কোথায় & হদে, হড়ে \\
\hline Big & ঊড় & ড-অঁর & Source of Money & টাকার উৎস & টেঁয়া হদেত্তুন হামায় \\
\hline Age & বয়স কত & হ বছর & Girls, Daughter & মেয়ে, কন্যা & মাইয়া ফোয়া \\
\hline Literacy & $\begin{array}{l}\text { কোন ক্লাস পর্যন্ত } \\
\text { পড়েছে }\end{array}$ & ফরনা হদ্দুর পজ্জ্যে & Did & করতো & গইত্ত্যু \\
\hline Myanmar & এায়ানমার & বর্মা/বার্মা & Came & এসেছে & আঁইইচ্চ্যু \\
\hline Why & কিসের জন্য & কিওল্ল্যায় & What happened & কি হয়েছিলো & কি অইলদে \\
\hline Asset & সম্পত্তি & সয় সম্পত্তি & Relative & আত্নীয় & গুস্তি, কুল \\
\hline $\begin{array}{l}\text { Marriage } \\
\text { Registration }\end{array}$ & $\begin{array}{l}\text { বিয়ের } \\
\text { রেজিস্ট্রেশন }\end{array}$ & $\begin{array}{l}\text { বিয়ার রেজিস্টি, হাগজ- } \\
\text { ফন্না }\end{array}$ & First Child & প্রথম বাচ্চা & ফইল্ল্যা ফোয়া \\
\hline Did you see & দেখেছেন কি & দেইখ্যুন না & Did you hear & শুনেছেন কি & উইন্যুন না \\
\hline $\begin{array}{l}\text { Whom they } \\
\text { marry }\end{array}$ & $\begin{array}{l}\text { কাদেরকে বিয়ে } \\
\text { করে }\end{array}$ & হারারে বিয়া গরে & Early Marriage & বাল্য বিবাহ & $\begin{array}{l}\text { অল্প বয়সত বিয়া, স- } \\
\text { রে বিয়া }\end{array}$ \\
\hline Polygamy & বহু বিবাহ & বউত্ বিয়া & Early Pregnancy & অকাল গর্ভধারণ & অল্প বয়সত বিয়া \\
\hline Negative & নেতিবাচক & হারাফ চোগত্ দেহে & Procedure & পদ্ধতি & হেন্দল্যা গরি, হেন গরি \\
\hline Our Society & আমাদের সমাজ & আরর্ কোমত্ & Young People & অল্প বয়সী & হম বয়সী \\
\hline Discourage & ডনরুৎসাহী & ন লইত চায় & Family Obligation & পারিবারিক বাধা & ঘরত মানা \\
\hline New form & নতুন কিছু & নোয়া কিসু & Lengthy Process & দীর্ঘ মেয়াদী & বহুত দিনর লায় \\
\hline Short Process & স্বল্প মেয়াদী & হম দিনর লায় & Sterilization & বন্ধ্যাত্ব করন & $\begin{array}{l}\text { এক্কেবারে ফোয়া ন } \\
\text { অইবারলায় }\end{array}$ \\
\hline MR & এমআর & ফোয়া ফেলিবার লায় & Maternal Health & মাতৃ স্বাস্থ্য & ফোয়াতির শরীর \\
\hline Governmental & সরকারী & হুকুমত, সরকারী & Example & উদাহারণ & মেসাল \\
\hline Health Centre & স্বাস্থ্যকেন্দ্র & ডাক্টর হানা & Child Delivery & $\begin{array}{l}\text { বাচ্চা ডেলিভারি } \\
\text { করায় }\end{array}$ & বাচ্চা ধরে, ফোয়া ধরে \\
\hline Help & সাহায্য করে & বল দেয় & Recent & সাম্প্রতিক & হয়েক দিন আগে \\
\hline Expensive & দামী & মঙ্গা & Majority & অনেকে & বহুত কিয়া \\
\hline Unmarried & অবিবাহিত & আবিয়াত্তা & Married & বিবাহিত & বিয়াত্তা \\
\hline $\operatorname{Sin}$ & ঈাপ & গুনাহ & Child Delivery & জন্মদানে সাহায্য & ফোয়া ধরা \\
\hline Rain fall & বৃষ্টি আসলে & ঝড় আইলে & All & সব & ব্যাক্কুন/বেক্ক্যুন \\
\hline $\begin{array}{l}\text { Male } \\
\text { Sterilization }\end{array}$ & ছেলে বন্ধ্যা করণ & ফোয়া হাসী গরণ & Pond & পুকুর & ফইর্ \\
\hline $\begin{array}{l}\text { When did you } \\
\text { come }\end{array}$ & কবে আসছো & হত্তে আইসসো & Tell me & আমাকে বলেন & অাঁরে হ-না \\
\hline
\end{tabular}




\begin{tabular}{|c|c|c|c|c|c|}
\hline Can't do & পারবো না & ন ফাইজ্জুম & From here & এখান থেকে & এদেত্তুন \\
\hline Get it & পেয়েছি & ফাই ই & Birth Control & জন্মানিয়ন্ত্রণ & আজাব দূরে গরন \\
\hline Getting older & বড় হয়েছে & হোঁইচ্চা অইয়ে & Reproductive & জন্মদান & জাত বাড়ন \\
\hline Needs & চাহিদা, প্রয়োজন & লাগে, জরুরত & Practice & চর্চা & আদত \\
\hline Dowry & যৌতুক & সামান, হাদিয়া & AIDS & এইডস্ & এ আই ডি এস \\
\hline Way/Resource & উপায় & তরিকা & Solve/Justice & সমাধান, বিচার & ইনসাফ \\
\hline Menstruation & মাসিক & $\begin{array}{l}\text { গোছল অইয়ে, ঘর } \\
\text { ঘইল্লে, জোয়ান অইয়ে }\end{array}$ & Violence & অত্যাচার & বল জোর গরা \\
\hline Risk & ঝুঁকি & মছিবত, ডর & Ignorance & অবহেলা & ইনকার \\
\hline Desire & ইচ্ছা & নিয়ত & Healer & কবিরাজ & বৈদ্য \\
\hline Rich & বড়লোক & তেয়োঙ্গ & Disease & রোগ & ব্যারাম \\
\hline STI & যৌনরোগ & ধাতু ব্যারাম & Prevent/Defense & প্রতিরোধ & রুখিবার লাই \\
\hline $\begin{array}{l}\text { Marriage } \\
\text { Registration }\end{array}$ & বিয়ের নিবন্ধন & লাতুং & Rights & অধিকার & হক \\
\hline Certificate & সনদ & গুন্ডু & Terrorist & উগ্রপন্থী, জঙ্গী & আলেকিন \\
\hline Implants & রোপন & রোয়া & Chairman & চেয়ারম্যান & হুক্কাডো \\
\hline Condom & কনডম & $\begin{array}{l}\text { ফটনা, ফদেনা, } \\
\text { কুইনডম, কন্টং }\end{array}$ & Rain & বৃষ্টি & বারিশ, বারিশা \\
\hline Shelter & আশ্রয় & সাহারা & Canal, Lake & খাল, বিল & ঢেপা, ঢেবা \\
\hline Adolescent & $\begin{array}{l}\text { ফ্যীবন (মেয়েদের } \\
\text { ক্ষেত্রে) }\end{array}$ & ঘর ঘলানো & Fire Woods & জালানী কাঠ & লাকড়ি \\
\hline Hill, Mountain & ঈাহাড় & মুরা, মুড়া & Pillar & ঘরের খুটি & হারাং \\
\hline Family hear & পরিবার প্রধান & উজি & Virus & ভাইরাস, জীবানু & পুক্, পুগ, \\
\hline Separate & $\begin{array}{l}\text { পরিবার থেকে } \\
\text { আলাদা }\end{array}$ & জুদা & Yaba & ইয়াবা & ডব্লিউ ওয়াই, ট্যাবলেট \\
\hline
\end{tabular}




\section{ANNEX 2: DATA COLLECTION INSTRUMENTS}

\section{In-depth interviews (IDIs) with Rohingya adolescents}

A total of 48 IDIs were conducted following a semi-structural interview guideline with married and unmarried Rohingya adolescent girls and boys aged 14-24 years. These IDIs explored their knowledge, attitudes, and practices relating to sexual and reproductive health, family planning, contraception, HIV/AIDS, STI, menstrual regulation, and their service seeking behavior. The study also explored other related issues, such as marriage practices and rituals, safety and security concerns, substance abuse, future aspirations and life after displacement from their homeland.

\section{In-depth interview (IDIs) with service providers and program personnel}

A total of 24 IDIs were conducted with service providers and program managers. Twelve IDIs were conducted with program personnel from national and international organizations providing SRH and health services. Among the 12 service providers, there were medical officers, paramedics, midwives, and medical assistants. Senior and midlevel program personnel from sexual and reproductive health (SRH) and gender-based-violence (GBV) focused programs were selected for interview. The main purpose of interviewing these two groups of people was to capture their experiences and insights about the current service delivery situation.

\section{FGDs with Majhees and Imams of the Rohingya community}

Three FGDs were conducted with community leaders (known as “Majhee") and three FGDs were conducted with religious leaders (known as "Imam"). Majhees and Imams in these settings are always male. The FGDs with these two groups were expected to provide insight for understanding community dynamics and the dominant community norms, practices, and attitudes related to marriage, family planning, $\mathrm{SRH}$, and other issues from which the social behavior and cultural practices of the Rohingya evolve. As potential gate-keepers, these influential Rohingya males are expected to be better able to reflect analytically about RH/FP beliefs and practices among the Rohingya, and to contrast practices in their homeland in Myanmar prior to displacement to practices in the camp. The findings from FGDs with Majhees and Imams have been described in different sections of the report under specific themes that emerged from the data.

\section{FGDs with adult women of Rohingya community}

Two FGDs were conducted with adult Rohingya women living in the camps to understand women's perspectives and attitudes towards family planning and contraceptive methods, marriage, fertility intentions and child bearing norms, and SRH-related behavior and knowledge. The FGDs explored collective norms and behaviors related to service seeking and availability of facilities for maternal and child health in the pre-arrival phases in Myanmar, as well as the perception of health services available in the camps for adolescent girls and women.

\section{FGDs with adolescent and youth from host community}

A total of 6 FGDs were organized with the adolescent and young girls and boys from the Bengali host community aged 18-24 years to understand the surrounding environment and current status of SRH and FP services, access, coverage, utilization, quality, and effectiveness for the host community. Among 6 FGDs, 2 with married girls, 2 with unmarried girls, 1 with married boys and 1 with unmarried boys were conducted in the host community. 



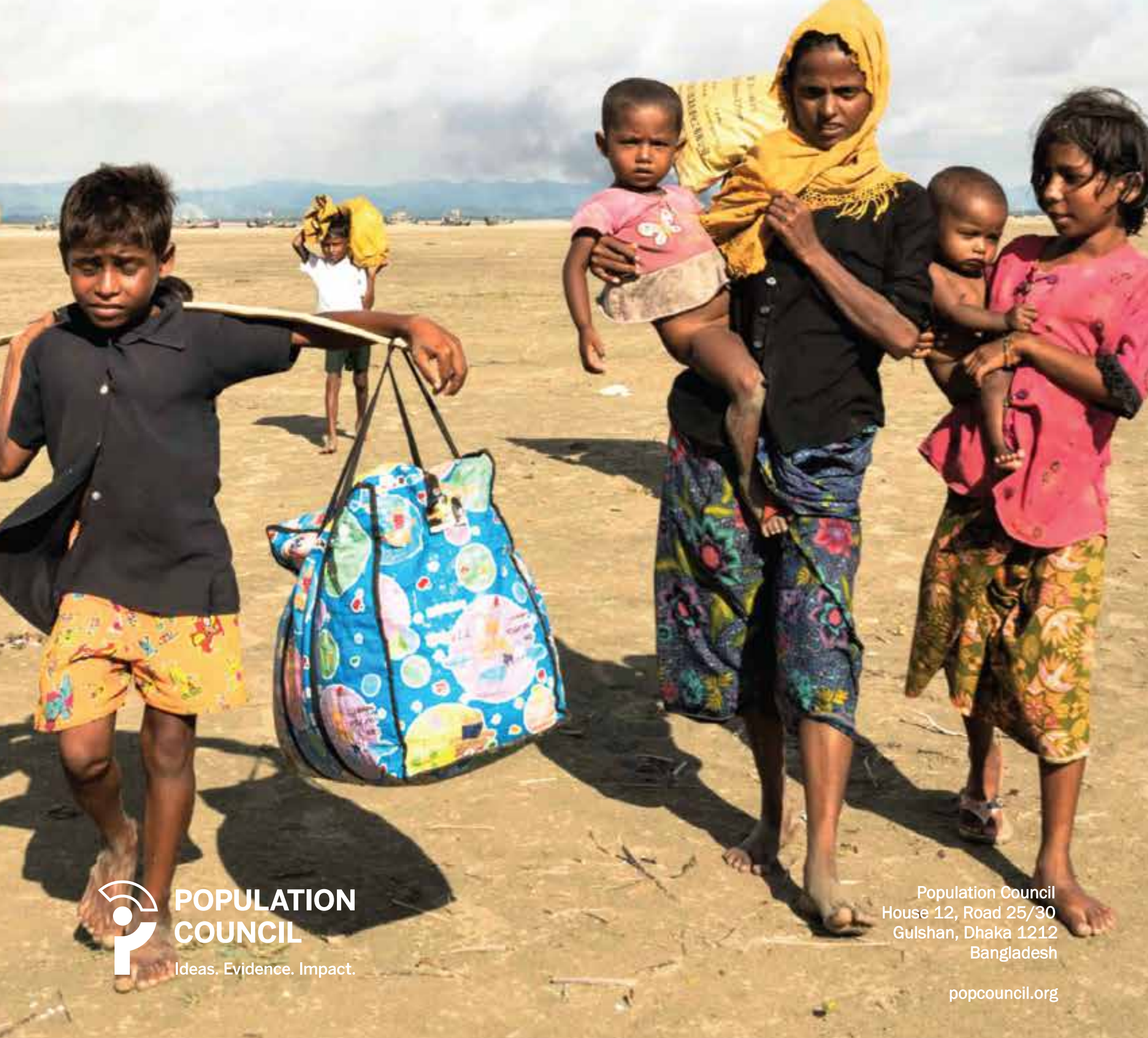

